Pacific

Journal of

Mathematics

CHARACTERIZATION OF HARDY SPACES BY SINGULAR INTEGRALS AND 'DIVERGENCE-FREE' WAVELETS

J.E. Gilbert, J.A. Hogan, And J.D. LAKey 


\title{
CHARACTERIZATION OF HARDY SPACES BY SINGULAR INTEGRALS AND 'DIVERGENCE-FREE' WAVELETS
}

\author{
J.E. Gilbert, J.A. Hogan, And J.D. Lakey
}

In memory of A. Uchiyama

\begin{abstract}
The Hardy space $H_{\rho_{r}}^{1}\left(\mathbb{R}^{n}\right)$ consists of all divergence free $r$-form distributions $f$ whose non-tangential maximal functions are in $L^{1}\left(\mathbb{R}^{n}\right)$. We say that a system of singular integrals characterizes $H_{\rho_{r}}^{1}\left(\mathbb{R}^{n}\right)$ if this space consists precisely of those divergence-free $r$-form distributions $f$ whose images under the singular integral operators are integrable. When the operators are determined by Fourier multipliers, necessary and sufficient conditions are prescribed on the multipliers in order that the system characterize $H_{\rho_{r}}^{1}\left(\mathbb{R}^{n}\right)$. The condition is analogous to the Janson-Uchiyama condition for the scalar-valued case and the characterization follows the lines of Uchiyama's constructive decomposition of BMO. In particular, it is shown how to build divergence-free $r$-form wavelets which play the same role that the $R$. Fefferman-Chang elementary decomposition played in Uchiyama's work.
\end{abstract}

\section{Introduction.}

In this paper we continue to develop real-variable theory for Hardy spaces $H_{\rho_{r}}^{p}\left(\mathbb{R}^{n}\right)$ of divergence-free, or, more precisely, 'co-closed' $r$-forms. Though these spaces arise as the 'real parts' of boundary values of $r+1$-form solutions of the Hodge-deRham $\left(d, d^{*}\right)$-system in the upper half-space $\mathbb{R}_{+}^{n+1}$, the most natural definition for them is a strictly real variable one using the non-tangential maximal function. A characterization by singular integrals then seeks an understanding of the relationship between the two possible definitions. The characterization given here is based on Uchiyama's constructive decomposition arguments, with a view towards broadening the scope of those arguments to other geometric settings.

Let $\mathcal{H}$ be a finite-dimensional real Hilbert space with inner product $\langle\cdot, \cdot\rangle$ and norm $\|\cdot\|_{\mathcal{H}}$. The real variable Hardy space $H^{p}\left(\mathbb{R}^{n}, \mathcal{H}\right), 0<p<\infty$, 
consists of those $\mathcal{H}$-valued distributions for which

$$
\|f\|_{H^{p}}=\left(\int_{\mathbb{R}^{n}}|\mathcal{N}(f)(x)|^{p} d x\right)^{1 / p}
$$

is finite where

$$
\mathcal{N}(f)(x)=\sup _{|x-y|<t}\|u(y, t)\|_{\mathcal{H}}
$$

is the non-tangential maximal function of the Poisson extension $u(x, t)$ of f. As is well-known, $H^{p}\left(\mathbb{R}^{n}, \mathcal{H}\right)=L^{p}\left(\mathbb{R}^{n}, \mathcal{H}\right)$ when $1<p<\infty$ and the notation $H^{p}, L^{p}$ will be used interchangably for such values of $p$. The usual Hardy $H^{p}\left(\mathbb{R}^{n}\right)$-spaces correspond to the scalar case $\mathcal{H}=\mathbb{R}$. For the most part in this paper $\mathcal{H}$ will be the $r^{t h}$-exterior power $\Lambda_{r}\left(\mathbb{R}^{n}\right)$ of $\mathbb{R}^{n}$, and we shall use the term divergence free $r$-form rather loosely to mean any function or distribution $f: \mathbb{R}^{n} \rightarrow \Lambda_{r}\left(\mathbb{R}^{n}\right)$ such that

$$
d^{*} f=\sum_{j=1}^{n} \mu_{j}^{*} \frac{\partial f}{\partial x_{j}}=0
$$

where $\mu_{j}^{*}$ denotes interior multiplication by the element $e_{j}$ in the standard basis for $\mathbb{R}^{n}$ (cf. Section 3 for more details on forms and their properties). By a distribution we shall always mean one which is restricted at infinity in the sense of Stein so that harmonic extensions and the action of singular integrals on such distributions are well-defined $([\mathbf{1 6}]$, p. 123). The divergence-free condition then singles out a closed subspace of $H^{1}\left(\mathbb{R}^{n}, \Lambda_{r}\left(\mathbb{R}^{n}\right)\right)$.

Definition 1.4. The Hardy space $H_{\rho_{r}}^{1}\left(\mathbb{R}^{n}\right)$ consists of all divergence free $r$-form distributions $f$ whose non-tangential maximal function $\mathcal{N}(f)$ is in $L^{1}\left(\mathbb{R}^{n}\right)$.

Alternatively, one can follow the Stein and Weiss formulation of Euclidean $H^{p}$-theory in the 1960's (e.g., [17], [18]), beginning with boundary values of a space of $r+1$-form solutions of the Hodge-deRham $\left(d, d^{*}\right)$-system on the upper half-space $\mathbb{R}_{+}^{n+1}$. Due to relations among their components these boundary values form a closed subspace of $L^{1}\left(\mathbb{R}^{n}, \Lambda_{r+1}\left(\mathbb{R}^{n+1}\right)\right)$. Indeed,

$$
f \longrightarrow e_{0} \wedge f+\sum_{j=1}^{n} e_{j} \wedge\left(R_{j} f\right)=(\mathcal{I}+\mathcal{R}) f
$$

defines a 1-1 mapping from $H_{\rho_{r}}^{1}\left(\mathbb{R}^{n}\right)$ onto this space of boundary values, turning $H_{\rho_{r}}^{1}\left(\mathbb{R}^{n}\right)$ into a space of 'real parts' of boundary values $([\mathbf{7}],[8])$. A divergence-free distribution $f$ then belongs to $H_{\rho_{r}}^{1}\left(\mathbb{R}^{n}\right)$ if and only if $(\mathcal{I}+$ $\mathcal{R}) f$ belongs to $L^{1}\left(\mathbb{R}^{n}, \Lambda_{r+1}\left(\mathbb{R}^{n+1}\right)\right)$. This definition emphasizes the relation between over-determinedness of the $\left(d, d^{*}\right)$-system and the 'divergence-free' or so-called 'gauge' condition on these 'real parts'; implicit also is the role of sub-harmonicity in arriving at the $L^{1}$-space of boundary values. 
A major advance in understanding $H^{1}$-spaces without regard to harmonicity occured when C. Fefferman and Stein characterized $H^{1}\left(\mathbb{R}^{n}\right)$ in terms of the grand maximal function ([5]). But could anything comparable be said about singular integrals? The need to understand the relationship between these two approaches - one requiring the non-degeneracy of various maximal functions, the other requiring non-degeneracy of a system of singular integrals - led C. Fefferman to raise the question of describing the families of singular integral operators that characterize $H^{1}([4])$. In the present context singular integral will mean a standard Calderón-Zygmund integral operator

$$
f(x) \longrightarrow \int_{\mathbb{R}^{n}}\langle\hat{f}(\xi), m(\xi)\rangle e^{2 \pi i x \cdot \xi} d \xi
$$

determined by its 'multiplier' $m=m(\xi)$, a $\Lambda_{r}\left(\mathbb{C}^{n}\right)$-valued $\mathcal{C}^{\infty}$-function which is homogeneous of degree 0 . The multiplier can thus be identified with a function $m=m(\omega)$ on the unit sphere $\Sigma_{n-1}$ and we shall refer to such an operator, or systems of such operators, as a singular integral operator having 'smooth symbol $m$ '; in the case of a system $\mathbb{K}=\left(\mathcal{K}_{1}, \ldots, \mathcal{K}_{M}\right)$ the symbol will be an operator $m=m(\omega): \Lambda_{r}\left(\mathbb{C}^{n}\right) \rightarrow \mathbb{C}^{M}$. The operator will be real if and only if $m(-\omega)=\overline{m(\omega)}, \omega \in \Sigma_{n-1}$. Fefferman's question now becomes:

Problem 1.6. Prescribe necessary and sufficient conditions for a system of singular integrals $\mathbb{K}=\left(\mathcal{K}_{1}, \ldots, \mathcal{K}_{M}\right)$ to characterize $H_{\rho_{r}}^{1}\left(\mathbb{R}^{n}\right)$ in the sense that $H_{\rho_{r}}^{1}\left(\mathbb{R}^{n}\right)$ consists precisely of those divergence-free $r$-form distributions $f$ for which $\mathbb{K} f \in L^{1}\left(\mathbb{R}^{n}, \mathbb{C}^{M}\right)$.

One might also hope that (1.6) provides further insight into the role of over-determinedness in (1.5) without regard to subharmonicity. On one hand, we can consider $H_{\rho_{r}}^{1}$ as a prototype of Hardy spaces of distributions satisfying some added geometric constraint - the divergence-free condition in this case - and our approach to problem (1.6) reflects this point of view. On the other hand, the precise geometric structure of the present case allows for a particularly simple solution as in the proof of Theorem A.

To arrive at a solution to (1.6) we interpret the divergence free condition on the Fourier transform side. The crucial idea is to use (1.3) to provide an orthogonal decomposition $\Lambda_{r}\left(\mathbb{C}^{n}\right)=N_{r}(\omega) \oplus T_{r}(\omega)$ of $\Lambda_{r}\left(\mathbb{C}^{n}\right)$ at each point $\omega$ of the unit sphere $\Sigma_{n-1}$ in $\mathbb{R}^{n}$, splitting $\Lambda_{r}\left(\mathbb{C}^{n}\right)$ into its respective 'normal' and 'tangential' components

$$
N_{r}(\omega)=\left\{a \in \Lambda_{r}\left(\mathbb{C}^{n}\right): \omega \wedge a=0\right\}, \quad T_{r}(\omega)=N_{r}(\omega)^{\perp}
$$

at $\omega$. This splitting corresponds to the Hodge Decomposition of $H^{1}\left(\mathbb{R}^{n}, \Lambda_{r}\right)$. 
Theorem A. A system of singular integrals $\mathbb{K}$ characterizes $H_{\rho_{r}}^{1}\left(\mathbb{R}^{n}\right)$ if and only if for each $\omega \in \Sigma_{n-1}$ its symbol $m(\omega)$ has the property

$$
\operatorname{rank}(m(\omega) \oplus m(-\omega))=2\left(\begin{array}{c}
n-1 \\
r
\end{array}\right)
$$

as a mapping $(a, b) \rightarrow m(\omega) a+m(-\omega) b$ from $T_{r}(\omega) \oplus T_{r}(-\omega)$ into $\mathbb{C}^{M}$.

The algebraic condition in Theorem A thus requires the restriction of $m(\omega) \oplus m(-\omega)$ to $T_{r}(\omega) \oplus T_{r}(-\omega)$ be 1-1 since

$$
\operatorname{dim}_{\mathbb{C}} N_{r}(\omega)=\left(\begin{array}{l}
n-1 \\
r-1
\end{array}\right), \quad \operatorname{dim}_{\mathbb{C}} T_{r}(\omega)=\left(\begin{array}{c}
n-1 \\
r
\end{array}\right) .
$$

It might be 1-1 on a larger subspace of $\Lambda_{r}\left(\mathbb{C}^{n}\right) \oplus \Lambda_{r}\left(\mathbb{C}^{n}\right)$, of course, but it is only the behaviour on $T_{r}(\omega) \oplus T_{r}(-\omega)$ that is of importance for characterizing $H_{\rho_{r}}^{1}\left(\mathbb{R}^{n}\right)$. For 0 -forms, $T_{0}(\omega)=\mathbb{C}$ and so in the case of complex-valued functions Theorem A reduces to the well-known result of Uchiyama and Janson $([\mathbf{1 0}],[\mathbf{1 9}])$. In the more general form-valued case Theorem A follows from the corresponding characterization of $H^{1}\left(\mathbb{R}^{n}, \Lambda_{r}\left(\mathbb{C}_{\tilde{\mathbb{K}}}^{n}\right)\right)$. To achieve this we augment the system $\mathbb{K}$ obtaining an auxiliary system $\tilde{\mathbb{K}}$ that characterizes $H^{1}\left(\mathbb{R}^{n}, \Lambda_{r}\left(\mathbb{C}^{n}\right)\right)$ and whose restriction to $H_{\rho_{r}}^{1}\left(\mathbb{R}^{n}\right)$ agrees with $\mathbb{K}$.

This simple proof does not capture the full import of Uchiyama's solution of problem (1.6), however, because he gave at the same time a constructive version of the C. Fefferman-Stein decomposition of $\mathrm{BMO}\left(\mathbb{R}^{n}\right)$. Indeed, Uchiyama showed that:

(i) the maximal rank condition on $m(\omega)$ provides an 'adaptive-pseudoinvertibility' criterion on $\mathbb{K}$, so that

(ii) for each $g \in \operatorname{BMO}\left(\mathbb{R}^{n}\right)$ one can construct inductively a function $h \in$ $L^{\infty}\left(\mathbb{R}^{n}, \mathbb{C}^{M}\right)$ with $\mathbb{K}^{*} h=g$.

The construction is carried out by applying an appropriate pseudoinverse to each term in the R. Fefferman-Chang elementary particle expansion of $g$. In fact, Uchiyama's argument is valid in any vector-valued BMO-type space so long as the functions possess an elementary particle expansion and the norm is determined by a Carleson condition on the coefficients in the expansion. More precisely, let $B$ be a closed subspace of $\operatorname{BMO}\left(\mathbb{R}^{n}, \mathcal{H}\right)$ where $\mathcal{H}$ is a finite-dimensional Hilbert space. Suppose furthermore,

(1.9)(a) for each dyadic cube $Q$ there is a function $\psi_{Q} \in B$ with $\operatorname{supp}\left(\psi_{Q}\right) \subseteq m Q, \quad \int_{\mathbb{R}^{n}} \psi_{Q}(x) d x=0, \quad\left|D^{\alpha} \psi_{Q}(x)\right| \leq$ const. $|Q|^{-1 / 2-|\alpha| / n}$, uniformly in $Q$ for all $0 \leq|\alpha| \leq 1$, 
(1.9)(b) each $g$ in $B$ has an expansion $g \sim \sum_{Q} c_{Q} \psi_{Q}$, converging in the sense of distributions, so that

$$
g \longrightarrow \sup _{Q}\left(\frac{1}{|Q|} \sum_{Q^{\prime} \subseteq Q}\left|c_{Q^{\prime}}\right|^{2}\right)^{1 / 2}
$$

provides an equivalent norm on $B$, and

$(1.9)(\mathrm{c})$ conversely, to each sequence $\left\{c_{Q}\right\}$ satisfying the Carleson condition of (1.9)(b) there corresponds a function $g \sim \sum_{Q} c_{Q} \psi_{Q}$.

The restriction to $B$ of any standard Calderón-Zygmund operator with symbol $m(\omega): \mathcal{H} \rightarrow \mathbb{C}^{M}$ will automatically map $B$ into $\operatorname{BMO}\left(\mathbb{R}^{n}, \mathbb{C}^{M}\right)$, and its adjoint will map $\operatorname{BMO}\left(\mathbb{R}^{n}, \mathbb{C}^{M}\right)$ into $\operatorname{BMO}\left(\mathbb{R}^{n}, \mathcal{H}\right)$. But this adjoint need not have range in $B$, of course, and the abstract formulation of Uchiyama's result has to reflect this fact. For convenience, it is stated here only for real systems.

Constructive Decomposition Theorem. Let $B$ be a subspace of $\mathrm{BMO}\left(\mathbb{R}^{n}, \mathcal{H}\right)$ satisfying $(1.9)$ and let $\mathbb{K}$ be a real standard singular integral system such that to every $\nu \in \Sigma_{M-1}$ there corresponds a system $\mathbb{L}_{\nu}$ for which

$$
\mathbb{K}^{*} \circ \mathbb{L}_{\nu}=I, \quad \nu \cdot \mathbb{L}_{\nu}=0
$$

as operators from $B$ into $\mathrm{BMO}\left(\mathbb{R}^{n}, \mathcal{H}\right)$ and $\mathrm{BMO}\left(\mathbb{R}^{n}, \mathbb{C}^{M}\right)$, respectively. Then for each $g$ in $B$ one can construct an $h$ in $L^{\infty}\left(\mathbb{R}^{n}, \mathbb{C}^{M}\right)$ and $f$ in $\operatorname{BMO}\left(\mathbb{R}^{n}, \mathcal{H}\right)$ so that $\mathbb{K}^{*} h+f=g$ and $\|h\|_{\infty} \leq$ const. $\|g\|_{B}$; furthermore, if $B$ can be identified with the dual space of a subspace of $H^{1}\left(\mathbb{R}^{n}, \mathcal{H}\right)$, then $\langle f, G\rangle=0$ for all $G$ in the pre-dual of $B$.

The detailed proof in the scalar case given by [1] carries over to the vectorvalued case by using techniques that Uchiyama showed us after he read an earlier draft of this paper [20]. Both the support and $C^{1}$ conditions on the $\psi_{Q}$ in (1.9), as well as the hypothesis that $\mathbb{K}$ is real, can be weakened at the expense of more technical book keeping. The term $f$ arises in the inductive construction because at each stage a nonlinear splitting in $L^{\infty}\left(\mathbb{R}^{n}, \mathbb{C}^{M}\right)$ is performed on terms of the form $\mathbb{L}_{\nu}\left(\psi_{Q}\right)$ that takes these terms outside $\mathbb{L}_{\nu}(B)$. When $B=\operatorname{BMO}\left(\mathbb{R}^{n}, \mathcal{H}\right)$, then $f$ is necessarily zero.

In the present setting, let $B=\mathrm{BMO}_{\rho_{r}}$ denote the dual space of $H_{\rho_{r}}^{1}$ (cf. (4.4)). The Hodge decomposition ensures that $H_{\rho_{r}}^{1}$ is a complemented subspace of $H^{1}\left(\mathbb{R}^{n}, \Lambda_{r}\right)$, and so in turn $B=\mathrm{BMO}_{\rho_{r}}$ is a complemented subspace of $\mathrm{BMO}\left(\mathbb{R}^{n}, \Lambda_{r}\right)$. In particular, $B$ can be identified with the dual of a subspace of $H^{1}\left(\mathbb{R}^{n}, \Lambda_{r}\right)$. 
Theorem B. If $\mathbb{K}$ satisfies the rank condition of Theorem A, then for each $g \in \mathrm{BMO}_{\rho_{r}}$ one can construct an $h \in L^{\infty}\left(\mathbb{R}^{n}, \mathbb{C}^{M}\right)$ with $\|h\|_{\infty} \leq C\|g\|_{\mathrm{BMO}_{\rho_{r}}}$ and $\left(\left.\mathbb{K}\right|_{H_{\rho_{r}}^{1}}\right)^{*} h=g$.

Since $\left.\mathbb{K}\right|_{H_{\rho_{r}}^{1}}=\mathbb{K} \circ\left(\mathcal{R}^{*} \mathcal{R}\right)$ (cf. (3.7)), the Constructive Decomposition theorem can be applied to $\left.\mathbb{K}\right|_{H_{\rho_{r}}^{1}}$. The term $f$ in that theorem is identified with zero as an element of the dual of $H_{\rho_{r}}^{1}$. Theorem B is thus a corollary of Uchiyama's Constructive Decomposition theorem once one produces the family $\psi_{Q}$ and verifies the existence of pseudoinverses $\mathbb{L}_{\nu}$ satisfying the pseudoinverse condition in the Constructive Decomposition theorem. The $\psi_{Q}$ that we construct will form a bi-orthogonal system of wavelets - refinements of the R. Fefferman-Chang 'elementary particles' which, in addition to satisfying (1.9), are divergence-free. (cf. Theorem $\mathrm{C}$ and Theorem 4.6). The properties of these wavelets are more convenient than crucial. For example, the images of standard wavelets under $\mathcal{R}^{*} \mathcal{R}$ would provide sufficient tools for the constructive decomposition, though they would not have compact support. But the wavelets that we construct should be of independent use; moreover, their tensor product structure also facilitates the proof of necessity of the rank condition in Theorem A.

After some preliminary sections, Theorem A is established in Section 5. The existence of operators $\mathbb{L}_{\nu}$ in the case $B=\mathrm{BMO}_{\rho_{r}}$ can be established along lines similar to those used in proving the sufficiency of the rank condition in Theorem $\mathrm{A}$, that is, by augmenting $\mathbb{K}$ to $\tilde{\mathbb{K}}$, finding corresponding $\tilde{\mathbb{L}}_{\nu}$, then restricting to $H_{\rho_{1}}^{1}$. However, in keeping with the point of view of the abstract Uchiyama theorem, we will give a more complicated proof of the pseudoinverse condition - one that can be followed for decomposition of spaces $B$ where the 'augmentation trick' could fail. The pseudoinverse criterion is established in Section 6. Theorem B will then follow immediately from the abstract Uchiyama theorem for the case $B=\mathrm{BMO}_{\rho_{r}}$. To keep technicalities to a minimum we only verify Theorem $A$ and the pseudoinverse criterion for real systems $\mathbb{K}$, although our arguments can be extended to prove Theorems A and B for complex $\mathbb{K}$. There are very important examples of $\mathbb{K}$ that do not preserve real-valued functions, see Section 2. In Section 7 the wavelets are constructed to complete the proof of Theorem B.

\section{One-form case, examples.}

The significance of the rank condition in Theorem A is most easily illustrated in the case of 1 -forms $f=\left(f_{1}, \ldots, f_{n}\right)$, i.e., $\mathbb{R}^{n}$-valued functions and distributions, where 'divergence-free' has its traditional meaning

$$
d^{*} f=\frac{\partial f_{1}}{\partial x_{1}}+\cdots+\frac{\partial f_{n}}{\partial x_{n}}=0 .
$$


The decomposition $\mathbb{C}^{n}=N_{1}(\omega) \oplus T_{1}(\omega)$ of $\mathbb{C}^{n}$ corresponds to the familiar splitting of any 1-form into its 'divergence' and 'curl-free' parts. It also lives up to the geometric terminology used:

$$
N_{1}(\omega)=\left\{a \in \mathbb{C}^{n}: a \wedge \omega=0\right\}=\mathbb{C} \omega
$$

can be identified with the complexification of the one-dimensional subspace of $\mathbb{R}^{n}$ passing through $\omega$, while the subspace

$$
T_{1}(\omega)=\left\{a \in \mathbb{C}^{n}: a \cdot \omega=0\right\}=N_{1}(\omega)^{\perp}
$$

orthogonal to $N_{1}(\omega)$ can be identified with the complexification of the tangent plane $\left\{a \in \mathbb{R}^{n}: a \cdot \omega=0\right\}$ to $\Sigma_{n-1}$ at $\omega$; in other words, $N_{1}(\omega)$ is the complexification of the normal line to this tangent plane.

2.3. Canonical Example. The symbol of the mapping (1.5) is given by

$$
m(\omega): a \longrightarrow e_{0} \wedge a-i \omega \wedge a \quad\left(a \in \mathbb{C}^{n}\right)
$$

where $\left\{e_{0}, \ldots, e_{n}\right\}$ is the extension of the standard basis of $\mathbb{R}^{n}$ to a basis for $\mathbb{R}^{n+1}$. Thus

$$
m(\omega) a+m(-\omega) b=e_{0} \wedge(a+b)-i \omega \wedge(a-b)=0 \quad\left(a, b \in \mathbb{C}^{n}\right)
$$

if and only if $a+b=0$ and $a-b \in N_{1}(\omega)$, i.e., $\operatorname{ker}(m(\omega) \oplus m(-\omega))=$ $\left\{(a,-a): a \in N_{1}(\omega)\right\}$. Hence the restriction of $m(\omega) \oplus m(-\omega)$ to $T_{1}(\omega) \oplus$ $T_{1}(-\omega)$ has maximal rank, so $\mathcal{I}+\mathcal{R}$ characterizes $H_{\rho_{1}}^{1}\left(\mathbb{R}^{n}\right)$.

2.4. Partition of Unity Example. Using spherical polar coordinates

$$
\omega=\left(\sin \theta \omega_{0}, \cos \theta\right), \quad \omega_{0} \in \Sigma_{n-2}, \quad 0 \leq \theta \leq \pi,
$$

for $\Sigma_{n-1}$, we can use Theorem A to show that each $f$ in $H_{\rho_{1}}^{1}\left(\mathbb{R}^{n}\right)$ is the sum of $2 n-1$ divergence-free 1 -forms each of which can be extended analytically to some tube domain. This is in complete analogy to Carleson's result for $H^{1}\left(\mathbb{R}^{n}\right)$, and, just as in Uchiyama's proof of that result, a partition of unity

$$
\phi_{1}(\omega)+\cdots+\phi_{2 n-1}(\omega)=1 \quad\left(\omega \in \Sigma_{n-1}\right)
$$

is required in which each $\phi_{j}$ is a real function having support in a portion of $\Sigma_{n-1}$ lying in some half-space (cf. [19], Corollary 3). The restriction on the support ensures that for each $\omega$ there exist $j, k$ such that

$$
\phi_{j}(\omega) \neq 0, \quad \phi_{j}(-\omega)=0 ; \quad \phi_{k}(\omega)=0, \quad \phi_{k}(-\omega) \neq 0 .
$$

Consequently, if

$$
m(\omega)=\left(\phi_{1}(\omega), \ldots, \phi_{2 n-1}(\omega)\right)
$$

then $m(\omega) \oplus m(-\omega)$ is $1-1$ from $T_{1}(\omega) \oplus T_{1}(-\omega)$ into $\mathbb{C}^{2 n-1}$, and so the corresponding $\mathbb{K}$ having $m=m(\omega)$ as symbol characterizes $H_{\rho_{1}}^{1}\left(\mathbb{R}^{n}\right)$. The connection of this analytic extension of $H_{\rho_{1}}^{1}\left(\mathbb{R}^{n}\right)$ functions with the functional calculus developed by McIntosh remains to be investigated ([12]). 
The construction of the $\phi_{j}$ proceeds by induction on $n$. For the unit circle $\Sigma_{1}$ there exist $\mathcal{C}^{\infty}$-functions forming a partition of unity $\phi_{1}+\phi_{2}+\phi_{3} \equiv 1$ where each function has support in some arc of length less than $\pi$. In passing to $\Sigma_{2}$, the previous three functions provide a partition of unity with respect to the longitudinal variable $\omega_{0}$, but some care must be taken with the latitudinal variable $\theta$ because of the ambiguity of spherical polar coordinates at the North and South Poles. Let $\phi_{+}=\phi_{+}(\theta)$ be an even $\mathcal{C}^{\infty}$ bump-function on $\mathbb{R}$ such that

$$
\phi_{+}(\theta)=0, \quad|\theta| \geq \theta_{0}, \quad \phi_{+}(\theta)=1, \quad|\theta| \leq \theta_{1}
$$

for some $0<\theta_{1}<\theta_{0}<\pi / 2$, and then define $\phi_{-}$by $\phi_{-}(\theta)=\phi_{+}(\pi-\theta)$. Finally, let $\phi=\phi(\theta)$ be a $\mathcal{C}^{\infty}$-function having support in $(0, \pi)$ so that $\phi_{+}+\phi+\phi_{-}$is a partition of unity on $[0, \pi]$. By construction,

$$
\phi_{+}(\theta)+\left\{\phi_{1}\left(\omega_{0}\right)+\phi_{2}\left(\omega_{0}\right)+\phi_{3}\left(\omega_{0}\right)\right\} \phi(\theta)+\phi_{-}(\theta)
$$

will then provide an unambiguously defined partition of unity by 5 functions on $\Sigma_{2}$ each of which has support in a portion of $\Sigma_{2}$ lying in some half-space. It is clear how to generalize this to any $\Sigma_{n-1}$.

2.5. Basis Example. The previous example can be refined to express each $f \in H_{\rho_{1}}^{1}\left(\mathbb{R}^{n}\right)$ as a sum $f=\sum_{j=1}^{2 n-1}\left(\sum_{k=1}^{n-1} f_{j k}\right)$ of basis functions each of which admits an analytic extension to some tube domain. We need a cross-section $\kappa: \Sigma_{n-1} \backslash\{\mathbf{- 1}\} \rightarrow S O_{n}$. With the notation of (2.4) set

$$
\kappa(\omega)=\left[\begin{array}{cc}
I-(1-\cos \theta) \omega_{0} \otimes \omega_{0} & -\sin \theta \omega_{0}^{t} \\
\sin \theta \omega_{0} & \cos \theta
\end{array}\right],
$$

regarding $\omega_{0}$ as a row matrix and its transpose $\omega_{0}^{t}$ as a column matrix. Under matrix multiplication on the right, 1. $\kappa(\omega)=\omega$; in addition, $\kappa(\omega)$ maps the tangent space $T_{1}(\mathbf{1}) \cong \mathbb{R}^{n-1}$ at the North Pole to the tangent space $T_{1}(\omega)$ at $\omega$. The image of the $\left\{e_{1}, \ldots, e_{n-1}\right\}$ for $T_{1}(\mathbf{1})$ will thus be a basis for $T_{1}(\omega)$, and we can use this together with the previous partition of unity to define a new symbol

$$
m(\omega)=\left[\begin{array}{cccc}
\phi_{1}(\omega) \kappa(\omega) e_{1} & \ldots & \phi_{2 n-2}(\omega) \kappa(\omega) e_{1} & \phi_{2 n-1}(\omega) \kappa(-\omega) e_{1} \\
\cdot & \ldots & \cdot & \cdot \\
\cdot & \ldots & \cdot & \cdot \\
\phi_{1}(\omega) \kappa(\omega) e_{n-1} & \ldots & \phi_{2 n-2}(\omega) \kappa(\omega) e_{n-1} & \phi_{2 n-1}(\omega) \kappa(-\omega) e_{n-1}
\end{array}\right],
$$

where it is assumed that $\phi_{2 n-1}=1$ in a neighborhood of the South Pole to ensure that $\kappa(-\omega)$ is well-defined. For the same reasons as before, $m(\omega) \oplus$ $m(-\omega)$ is $1-1$ from $T_{1}(\omega) \oplus T_{1}(-\omega)$ into $\mathbb{C}^{(n-1) \times(2 n-1)}$, and so again the corresponding $\mathbb{K}$ characterizes $H_{\rho_{1}}^{1}\left(\mathbb{R}^{n}\right)$. Notice that $m(\omega) \oplus m(-\omega)$ will not 
be 1-1 on all of $\mathbb{C}^{n} \oplus \mathbb{C}^{n}$. This example should also be contrasted with the wavelet representation of $f$ given later in (4.6).

2.5'. Normal Space Example. Let $H_{\tilde{\rho}_{1}}^{1}$ denote the complement of $H_{\rho_{1}}^{1}$, that is, the image of $\mathcal{R} \mathcal{R}^{*}$, inside of $H^{1}\left(\mathbb{R}^{n}, \mathbb{R}^{n}\right)$. Just as above, we can construct a symbol

$$
n(\omega)=\left[\begin{array}{llll}
\phi_{1}(\omega) \kappa(\omega) e_{n} & \ldots & \phi_{2 n-2}(\omega) \kappa(\omega) e_{n} & \phi_{2 n-1}(\omega) \kappa(-\omega) e_{n}
\end{array}\right] .
$$

Then $n(\omega) \oplus n(-\omega)$ will be $1-1$ on $N_{1}(\omega) \oplus N_{1}(-\omega)$ into $\mathbb{C}^{1 \times(2 n-1)}$, and so the same principles imply that the corresponding system $\mathbb{N}$ will characterize $H_{\tilde{\rho}_{1}}^{1}\left(\mathbb{R}^{n}\right)$. This example can readily be generalized to a system $\mathbb{N}_{r}$ characterizing $H_{\tilde{\rho}_{r}}^{1}$. Crucial to the proof of Theorem A will be the fact that for any $f \in H_{\rho_{r}}^{1}$, one has $\mathbb{N}_{r} f=0$.

2.6. Failure of Projection Examples. As an operator on $\mathbb{C}^{n}$ the symbol of the operator $\mathcal{R}^{*} \mathcal{R}$ has the property

$$
\langle m(\omega) a, b\rangle=\sum_{j, k} \omega_{j} \omega_{k} a_{j} \bar{b}_{k}
$$

so $m(\omega) a+m(-\omega)(-a)=0$ for any $a \in \mathbb{C}^{n}$. Thus the Hodge projection $\mathcal{R}^{*} \mathcal{R}$ does not characterize $H_{\rho_{1}}^{1}\left(\mathbb{R}^{n}\right)$. Similarly, neither of $I+\mathcal{R}^{*} \mathcal{R}, I+\mathcal{R} \mathcal{R}^{*}$ characterizes $H_{\rho_{1}}^{1}\left(\mathbb{R}^{n}\right)$. These last two examples are analogous to the first 'nontrivial' scalar-valued example $\{I, \mathcal{R} \cdot \mathcal{R}\}$ which failed to characterize $H^{1}\left(\mathbb{R}^{n}\right)$ (cf. [6]).

\section{Hodge decomposition, $r$-forms.}

To facilitate reading of the paper it may be helpful to recall some known though not always familiar algebraic ideas ([9], pp. 49-65). The exterior algebra

$$
\Lambda_{*}\left(\mathbb{R}^{n}\right)=\oplus \sum_{r=0}^{n} \Lambda_{r}\left(\mathbb{R}^{n}\right), \quad \Lambda_{0}\left(\mathbb{R}^{n}\right)=\mathbb{R}, \quad \Lambda_{1}\left(\mathbb{R}^{n}\right)=\mathbb{R}^{n}, \ldots
$$

associated with $\mathbb{R}^{n}$ is the algebra of linear combinations of wedge products $v_{1} \wedge \cdots \wedge v_{r}$ of vectors in $\mathbb{R}^{n}$. Wedge products

(3.2) $e_{\alpha}=e_{i_{1}} \wedge \ldots \wedge e_{i_{r}}$

$$
\alpha \in \mathcal{P}_{r}=\left\{\left(i_{1}, \ldots, i_{r}\right): 1 \leq i_{1}<\cdots<i_{r} \leq n\right\}
$$

of the standard basis $\left\{e_{1}, \ldots, e_{n}\right\}$ for $\mathbb{R}^{n}$ provide a basis for $\Lambda_{r}\left(\mathbb{R}^{n}\right)$; by convention, $e_{\emptyset}=1$ in the case $r=0$ when $\alpha$ is then the empty set $\emptyset$ and $\Lambda_{0}\left(\mathbb{R}^{n}\right)=\mathbb{R}$. The standard basis for $\mathbb{R}^{n}$ also extends to a standard basis $\left\{e_{0}, e_{1}, \ldots, e_{n}\right\}$ for $\mathbb{R}^{n+1}$. There is a canonical inner product $\langle\cdot, \cdot\rangle$ on $\Lambda_{r}\left(\mathbb{R}^{n}\right)$ with respect to which it is a real Hilbert space of dimension $\left(\begin{array}{l}n \\ r\end{array}\right)$ having $\left\{e_{\alpha}: \alpha \in \mathcal{P}_{r}\right\}$ as an orthonormal basis; $\Lambda_{*}\left(\mathbb{R}^{n}\right)$ is then a sum of 
Hilbert spaces, hence a real Hilbert space itself. To simplify notation we shall often write $\Lambda_{r}, \Lambda_{*}$ instead of $\Lambda_{r}\left(\mathbb{R}^{n}\right), \Lambda_{*}\left(\mathbb{R}^{n}\right)$. When Fourier transforms arise the corresponding complex exterior algebra $\Lambda_{*}\left(\mathbb{C}^{n}\right)$ along with its inner product structure will be needed, but in such cases full notation will always be retained to avoid any confusion.

The inner product structure on $\Lambda_{*}$ permits the introduction of Banach spaces of $\Lambda_{*}$-valued functions. For instance, the Lebesgue space of all squareintegrable $r$-forms

$$
f: \mathbb{R}^{n} \longrightarrow \Lambda_{r}, \quad f(x)=\sum_{\alpha \in \mathcal{P}_{r}} f_{\alpha}(x) e_{\alpha}, \quad(0 \leq r \leq n)
$$

on $\mathbb{R}^{n}$ will be denoted by $L^{2}\left(\mathbb{R}^{n}, \Lambda_{r}\right)$; similarly, $L^{2}\left(\mathbb{R}^{n}, \Lambda_{*}\right)$ will denote the space of all square integrable forms without restriction on $r$. In both cases the inner product is given by

$$
\langle f, g\rangle=\int_{\mathbb{R}^{n}}\langle f(y), g(y)\rangle d y
$$

with respect to the inner product on $\Lambda_{*}$. Many operators on forms exploit the algebra structure on $\Lambda_{*}$ : The wedge product $a \rightarrow e_{j} \wedge a$ defines the exterior multiplication operator $\mu_{j}: \Lambda_{*} \rightarrow \Lambda_{*}$ mapping $\Lambda_{r}$ to $\Lambda_{r+1}, 0 \leq r<$ $n$; its Hilbert space adjoint is the interior multiplication operator $\mu_{j}^{*}: \Lambda_{*} \rightarrow$ $\Lambda_{*}$ mapping $\Lambda_{r+1}$ to $\Lambda_{r}, 0 \leq r<n$. Direct computation shows that

$$
\mu_{j} \mu_{k}+\mu_{k} \mu_{j}=0 ; \quad \mu_{j}^{*} \mu_{k}^{*}+\mu_{k}^{*} \mu_{j}^{*}=0 ; \quad \mu_{j}^{*} \mu_{k}+\mu_{k} \mu_{j}^{*}=\delta_{j k} I .
$$

The exterior and interior Riesz transform operators

$$
\mathcal{R}=\sum_{k=1}^{n} \mu_{k} R_{k} ; \quad \mathcal{R}^{*}=-\sum_{k=1}^{n} \mu_{k}^{*} R_{k},
$$

thus define bounded operators on $L^{2}\left(\mathbb{R}^{n}, \Lambda_{*}\right)$ such that

$$
\langle\mathcal{R} f, g\rangle=\int_{\mathbb{R}^{n}}\langle\mathcal{R} f(x), g(x)\rangle d x=\int_{\mathbb{R}^{n}}\left\langle f(x), \mathcal{R}^{*} g(x)\right\rangle d x=\left\langle f, \mathcal{R}^{*} g\right\rangle .
$$

In view of (3.4),

$$
\mathcal{R} \mathcal{R}=0, \quad \mathcal{R}^{*} \mathcal{R}^{*}=0 ; \quad \mathcal{R} \mathcal{R}^{*}+\mathcal{R}^{*} \mathcal{R}=I
$$

since $\sum_{k} R_{k}^{2}=-I$. An immediate consequence of this is a crucial splitting of $L^{2}\left(\mathbb{R}^{n}, \Lambda_{r}\right)$.

Theorem 3.8 ([7] Hodge decomposition). The operators $\mathcal{R}^{*}$ and $\mathcal{R}^{*} \mathcal{R}$ define orthogonal projections from $L^{2}\left(\mathbb{R}^{n}, \Lambda_{r}\right)$ onto

$$
\left\{f \in L^{2}\left(\mathbb{R}^{n}, \Lambda_{r}\right): \mathcal{R} f=0\right\} \quad \text { and } \quad\left\{f \in L^{2}\left(\mathbb{R}^{n}, \Lambda_{r}\right): \mathcal{R}^{*} f=0\right\}
$$

respectively for each $r=0,1, \ldots, n$. 
The relation of this to the standard decomposition in terms of the HodgedeRham $\left(d, d^{*}\right)$-system on $\mathbb{R}^{n}$ is clear from the fact that

$$
\mathcal{R}=(-\Delta)^{-1 / 2} \circ d ; \quad \mathcal{R}^{*}=(-\Delta)^{-1 / 2} \circ d^{*} .
$$

In particular, we shall make frequent use of the fact that the massless condition $\mathcal{R}^{*} f=0$ and the divergence-free condition $d^{*} f=0$ are equivalent in the sense of distributions modulo polynomials $([8])$. For future reference it will also be important to note that all of these properties will persist in any space of forms on which Riesz transforms are well-defined and bounded.

\section{Wavelet analogues.}

Wavelets give a precise analogue of the R. Fefferman-Chang elementary particle decomposition that played a fundamental role in the constructive decomposition of BMO as described by Uchiyama and Christ-Geller. Better yet, divergence-free wavelets are now available thanks to independent work of Lemarié and Battle in the $r=1$ case. Our construction of divergencefree $r$-form wavelets in Section 7 is suggested, in turn, by a splitting of the index set $\mathcal{P}_{r}$ in (3.2) analogous with the splitting (1.7) of $\Lambda_{r}\left(\mathbb{C}^{n}\right)$. Here we will describe the wavelets, their role in norming $H_{\rho_{r}}^{1}$ and its dual, and their convenience in describing some Calderón-Zygmund operators.

Denote by $E^{*}$ the family of $\left(2^{n}-1\right)$ binary $n$-tuples $\epsilon=\left(\epsilon_{1}, \ldots, \epsilon_{n}\right)$ in $\{0,1\}^{n} \backslash(0, \ldots, 0) . E^{*}$ is sufficient to parametrize the mother wavelets needed in the real-valued case, i.e., when $r=0$ ([3], p. 317; [13], p. 84). Indeed, there exist real-valued scaling functions $\phi=\phi(x)$ with associated compactly supported mother wavelets $\left\{\psi_{\epsilon}: \epsilon \in E^{*}\right\}$ such that the family of usual translates and dilates

$$
\psi_{\epsilon, Q}(x)=2^{n j / 2} \psi_{\epsilon}\left(2^{j} x-k\right) \quad\left(Q=Q_{j k}, \epsilon \in E^{*}\right)
$$

of all these wavelets provide an orthonormal basis for $L^{2}\left(\mathbb{R}^{n}\right)$. The index $Q=Q_{j k} \in \mathcal{Q}_{j} \subset \mathcal{Q}$ denotes the dyadic cube of sidelength $l(Q)=2^{-j}$ with vertex at $k / 2^{j}$. Any such basis extends componentwise to the vector-valued case.

Let $\left\{e_{\alpha}\right\}_{\alpha \in \mathcal{P}_{r}}$ denote the standard basis for $\Lambda_{r}$. Then $\left\{\psi_{\epsilon} \otimes e_{\alpha}: \epsilon \in E^{*}, \alpha \in\right.$ $\left.\mathcal{P}_{r}\right\}$ is a family of $\left(\operatorname{card} E^{*}\right)\left(\operatorname{card} \mathcal{P}_{r}\right)$ mother wavelets whose translates and dilates provide an orthonormal basis of compactly supported functions for $L^{2}\left(\mathbb{R}^{n}, \Lambda_{r}\right)$. Because $\mathcal{R}^{*} \mathcal{R}$ is a singular operator, the divergence free images $\mathcal{R}^{*} \mathcal{R}\left(\psi_{\epsilon} \otimes e_{\alpha}\right)$ need not be compactly supported elements of $H_{\rho_{r}}^{2}\left(\mathbb{R}^{n}\right)$, however. In fact, for the $r=1$ case Lemarié has shown that there does not exist an orthonormal basis of compactly supported wavelets for $H_{\rho_{1}}^{2}\left(\mathbb{R}^{n}\right)$, though it is possible to build bi-orthogonal wavelets where the reconstructing wavelets are divergence-free, but no such constraint is imposed on the analyzing wavelets $([\mathbf{1 1}])$. Thus the price one pays for local properties in 
the case $r>0$ is a loss of orthogonality, but biorthogonality is more than sufficient for the present purpose.

For each $\epsilon \in E^{*}$ set

$$
i_{\epsilon}=\min \left\{i: \epsilon_{i}=1\right\} \quad\left(\epsilon \in E^{*}\right) .
$$

The index set $\mathcal{P}_{r}$ of $\Lambda_{r}\left(\mathbb{C}^{n}\right)$ can be split into two parts

$$
\mathcal{T}_{r}=\left\{\alpha: i_{\epsilon} \notin \alpha\right\}, \quad \mathcal{N}_{r}=\left\{\alpha: i_{\epsilon} \in \alpha\right\}
$$

analogous to the splitting (1.7) of $\Lambda_{r}\left(\mathbb{C}^{n}\right)$. Note that

$$
\operatorname{dim}_{\mathbb{C}} N_{r}(\xi)=\operatorname{card} \mathcal{N}_{r}, \quad \operatorname{dim}_{\mathbb{C}} T_{r}(\xi)=\operatorname{card} \mathcal{T}_{r},
$$

independent of $\epsilon \in E^{*}$. In Section 7 we shall prove the following result.

Theorem C. For each $r, 1 \leq r<n$, and each integer $m>0$ there is a family of compactly supported $r$-forms $\left\{\left(\psi^{\epsilon, \alpha}, \eta^{\epsilon, \alpha}\right): \epsilon \in E^{*}, \alpha \in \mathcal{T}_{r}\right\}$ having continuous derivatives as well as vanishing moments up to order $m$; furthermore,

(i) $\psi^{\epsilon, \alpha}$ is divergence-free and $\left\{\left(\psi^{\epsilon, \alpha}, \eta^{\epsilon, \alpha}\right): \epsilon \in E^{*}, \alpha \in \mathcal{T}_{r}\right\}$ is bi-orthogonal in the sense that

$$
d^{*} \psi^{\epsilon, \alpha}=0, \quad\left\langle\psi_{Q}^{\epsilon, \alpha}, \eta_{Q^{\prime}}^{\epsilon^{\prime}, \alpha^{\prime}}\right\rangle=\delta_{\epsilon \epsilon^{\prime}} \delta_{\alpha \alpha^{\prime}} \delta_{Q Q^{\prime}},
$$

hold, while

(ii) for $1 \leq p<\infty$, the mapping

$$
f \longrightarrow \mathcal{R}^{*} \mathcal{R} f(x)=\sum_{\epsilon, Q} \sum_{\alpha \in \mathcal{T}_{r}}\left\langle f, \eta_{Q}^{\epsilon, \alpha}\right\rangle \psi_{Q}^{\epsilon, \alpha}(x)
$$

is a projection from $H^{p}\left(\mathbb{R}^{n}, \Lambda_{r}\right)$ onto $H_{\rho_{r}}^{p}\left(\mathbb{R}^{n}\right)$.

These wavelets furnish convenient equivalent norms on $H_{\rho_{r}}^{1}\left(\mathbb{R}^{n}\right)$ and its dual space $\mathrm{BMO}_{\rho_{r}}\left(\mathbb{R}^{n}\right)$. The latter is the quotient space of $\mathrm{BMO}\left(\mathbb{R}^{n}, \Lambda_{r}\right)$ having norm defined by induction on $r$ :

$$
\|g\|_{\text {BMO }}=\sup _{Q}\left\{\inf _{h \in \mathrm{BMO}_{\rho_{r-1}}}\left(\frac{1}{|Q|} \int_{Q}|g(x)-\mathcal{R} h(x)|^{2} d x\right)^{\frac{1}{2}}\right\},
$$

the supremum being taken over all cubes in $\mathbb{R}^{n}$ having sides parallel to the coordinate axes (cf., [8]). In the case of 0 -forms it reduces to the usual $B M O$-space of real-valued functions for which

$$
\sup _{Q}\left\{\inf _{c \in \mathbb{R}}\left(\frac{1}{|Q|} \int_{Q}|h(x)-c|^{2} d x\right)^{1 / 2}\right\}
$$

is finite. 


\section{Theorem 4.6 ([8]).}

(i) Each $f \in H_{\rho_{r}}^{1}\left(\mathbb{R}^{n}\right)$ has a wavelet expansion

$$
f(x)=\sum_{\epsilon, Q} \sum_{\alpha \in \mathcal{T}_{r}}\left\langle f, \eta_{Q}^{\epsilon, \alpha}\right\rangle \psi_{Q}^{\epsilon, \alpha}
$$

whose square function norm

$$
\|\mathcal{S}(f)\|_{L^{1}}=\int_{\mathbb{R}^{n}}\left(\sum_{\epsilon, Q} \sum_{\alpha \in \mathcal{T}_{r}}\left|\left\langle f, \eta_{Q}^{\epsilon, \alpha}\right\rangle\right|^{2}\left|\psi_{Q}^{\epsilon, \alpha}(x)\right|^{2}\right)^{1 / 2} d x
$$

is equivalent to the non-tangential maximal norm (1.1) on $H_{\rho_{r}}^{1}\left(\mathbb{R}^{n}\right)$.

(ii) Each $g \in \mathrm{BMO}_{\rho_{r}}\left(\mathbb{R}^{n}\right)$ has a weak ${ }^{*}$-convergent expansion

$$
g(x)=\sum_{\epsilon, Q} \sum_{\alpha \in \mathcal{T}_{r}} c_{Q}^{\epsilon, \alpha} \psi_{Q}^{\epsilon, \alpha}
$$

for which the Carleson condition

$$
\sup _{Q}\left(\frac{1}{|Q|} \sum_{Q^{\prime} \subseteq Q \in \mathcal{Q}}\left|c_{Q^{\prime}}^{\epsilon, \alpha}\right|^{2}\right)^{1 / 2}<\infty
$$

defines a norm on $\mathrm{BMO}_{\rho_{r}}\left(\mathbb{R}^{n}\right)$ equivalent to (4.4). Conversely, any such sequence $\left\{c_{Q}^{\epsilon, \alpha}\right\}$ gives rise to such an element $g \in \mathrm{BMO}_{\rho_{r}}\left(\mathbb{R}^{n}\right)$.

This result follows from tent space arguments based on the fact that Theorem $\mathrm{C}$ provides a discrete resolution of the operator $\mathcal{R}^{*} \mathcal{R}$. In fact, similar arguments establish boundedness results for a large family of discrete sum operators

$$
\mathbb{D}: f \longrightarrow \sum_{\epsilon, Q} \sum_{\alpha \in \mathcal{T}_{r}}|Q|^{-1 / 2}\left\langle f, \eta_{Q}^{\epsilon, \alpha}\right\rangle \Psi_{Q}^{\epsilon, \alpha}(x)
$$

where each $\Psi_{Q}^{\epsilon, \alpha}$ is a $\mathbb{C}^{M}$-valued function which is assumed to have a vanishing moment as well as satisfy 'standard' size and smoothness conditions, cf., [7]. Indeed, this observation plays a crucial role in the constructive decomposition of $\mathrm{BMO}_{\rho_{r}}$, where $\Psi_{Q}^{\epsilon, \alpha}$ is the image of $\psi_{Q}^{\epsilon, \alpha}$ under a pseudoinverse $\mathbb{L}_{\nu_{Q}}$ of $\mathbb{K}$ as described in Section 6 .

The wavelet expansions in (4.6) are particularly useful in computing restriction results. Let $\mathbf{1}=(0, \ldots, 0,1)$ be the North Pole in $\Sigma_{n-1}$ and set

$$
P_{\mathbf{1}}: f \longrightarrow P_{\mathbf{1}} f(t)=\int_{\mathbb{R}^{n-1}} f(y+t \mathbf{1}) d y=\int_{-\infty}^{\infty} e^{2 \pi i s t} \hat{f}(s \mathbf{1}) d s \quad(t \in \mathbb{R}) .
$$

Formally, this is given on the transform side as the restriction to the line through the North Pole. Basic to the proof of necessity of the rank condition in Theorem $\mathrm{A}$ is the identification of the image of $H_{\rho_{r}}^{1}\left(\mathbb{R}^{n}\right)$ under $P_{\mathbf{1}}$. 
Theorem 4.8. The restriction $P_{\mathbf{1}}$ is bounded from $H_{\rho_{r}}^{1}\left(\mathbb{R}^{n}\right)$ onto $H^{1}\left(\mathbb{R}, \Lambda_{r}\left(\mathbb{R}^{n-1}\right)\right)$.

By the closed graph theorem therefore,

$$
\left\|P_{\mathbf{1}} f\right\|_{H^{1}}=\inf \left\{\|g\|_{H^{1}}: g \in H_{\rho_{r}}^{1}\left(\mathbb{R}^{n}\right): P_{\mathbf{1}} g=P_{\mathbf{1}} f\right\} .
$$

The corresponding result for $L^{1}\left(\mathbb{R}^{n}, \mathbb{R}^{M}\right)$ is well-known (cf. [15]). A proof of (4.8) appears at the end of Section 7.

\section{Proof of Theorem A.}

In what follows we shall assume that $\mathbb{K}$ is real. Proof of necessity of the maximal rank condition in Theorem A exploits some of the same ideas as Janson's proof in the scalar-valued case by reducing the setting to any onedimensional subspace of $\mathbb{R}^{n}$ which after rotation we can always assume is $\mathbb{R} \mathbf{1}$. The restriction of $\mathbb{K}$ to this line defines a singular integral

$$
\mathbb{K}_{\mathbf{1}}: h \longrightarrow \int_{\mathbb{R}} e^{2 \pi i s t} m(s \mathbf{1}) \hat{h}(s) d s=m(\mathbf{1})(h-i \tilde{h})+m(-\mathbf{1})(h+i \tilde{h}),
$$

where $\tilde{h}$ is the Hilbert transform of $h$. Furthermore, $\mathbb{K}_{\mathbf{1}}$ commutes with $P_{\mathbf{1}}$ in the sense that $\mathbb{K}_{1} \circ P_{1}=P_{1} \circ \mathbb{K}$.

Proof of necessity. Now suppose that $\mathbb{K}$ characterizes $H_{\rho_{r}}^{1}\left(\mathbb{R}^{n}\right)$. By the closed graph theorem, therefore,

$$
A_{0}\|f\|_{H^{1}} \leq\left\|\mathcal{K}_{1} f\right\|_{L^{1}}+\cdots+\left\|\mathcal{K}_{M} f\right\|_{L^{1}} \quad\left(f \in H_{\rho_{r}}^{1}\left(\mathbb{R}^{n}\right)\right)
$$

for some constant $A_{0}>0$. Using (4.8) we shall prove that

$$
\|h\|_{H^{1}} \leq \text { const. }\left\|\mathbb{K}_{\mathbf{1}} h\right\|_{L^{1}}
$$

then holds for all $h \in H^{1}\left(\mathbb{R}, \Lambda_{r}\left(\mathbb{R}^{n-1}\right)\right)$. Since $\mathbb{K}$, and hence $\mathbb{K}_{\mathbf{1}}$ is real, (5.3) will continue to hold for all $H^{1}$-functions $h: \mathbb{R} \rightarrow \Lambda_{r}\left(\mathbb{C}^{n-1}\right)$ as well, enabling us to apply it also to $h-i \tilde{h}, h+i \tilde{h}$. From this the necessity of the maximal rank condition follows quickly.

First we establish (5.3). By (4.9) the inequality

$$
A\left\|P_{\mathbf{1}} f\right\|_{H^{1}} \leq \inf _{g}\left\{\left\|\mathcal{K}_{1} g\right\|_{L^{1}}+\cdots+\left\|\mathcal{K}_{M} g\right\|_{L^{1}}\right\},
$$

holds after restriction of (5.2) to $\mathbb{R} \mathbf{1}$, the infimum being taken over $g \in$ $H_{\rho_{r}}^{1}\left(\mathbb{R}^{n}\right)$ with $P_{\mathbf{1}} g=P_{\mathbf{1}} f$. To proceed further we need a slightly stronger way of describing the norm of the $\mathcal{K}_{j} g([15]$, p. 115). Observe first that convolution

$$
(\tau * f)(x)=\int_{\mathbb{R}^{n-1}} \tau(v) f(x-v) d v \quad\left(f \in L^{1}\left(\mathbb{R}^{n}, \mathbb{R}^{M}\right)\right)
$$


by any real-valued function $\tau$ in $L^{1}\left(\mathbb{R}^{n-1}\right)$ can be defined componentwise; furthermore, $\tau * f$ belongs to $L^{1}\left(\mathbb{R}^{n}, \mathbb{R}^{M}\right)$ and

$$
P_{\mathbf{1}}: \tau * f \longrightarrow \int_{\mathbb{R}^{n-1}}\left(\int_{\mathbb{R}^{n-1}} \tau(v) f(y+t \mathbf{1}-v) d v\right) d y=\hat{\tau}(0)\left(P_{\mathbf{1}} f\right)(t) .
$$

In particular, $P_{\mathbf{1}}(\tau * f)=P_{\mathbf{1}} f$ whenever $\hat{\tau}(0)=1$.

Lemma 5.5. For each $f=\left(f_{1}, \ldots, f_{M}\right)$ in $L^{1}\left(\mathbb{R}^{n}, \mathbb{R}^{M}\right)$

$$
\int_{\mathbb{R}}\left\|P_{1} f(t)\right\|_{\mathbb{R}^{M}} d t=\inf _{\tau}\left(\int_{\mathbb{R}^{n}}\left|\left(\tau * f_{1}\right)(x)\right| d x+\cdots+\int_{\mathbb{R}^{n}}\left|\left(\tau * f_{M}\right)(x)\right| d x\right),
$$

the infimum being taken over all $\tau \in L^{1}\left(\mathbb{R}^{n-1}\right), \hat{\tau}(0)=1$.

Proof of Lemma 5.5. Obviously

$$
\int_{\mathbb{R}}\left\|P_{1} f(t)\right\|_{\mathbb{R}^{M}} d t \leq \int_{\mathbb{R}^{n}}\left|\left(\tau * f_{1}\right)(x)\right| d x+\cdots+\int_{\mathbb{R}^{n}}\left|\left(\tau * f_{M}\right)(x)\right| d x .
$$

Conversely, suppose the $f_{j}$ are real-valued continuous functions all of which have support in a compact subset, say $K$, of $\mathbb{R}^{n}$; in particular, $\left\|f_{j}\right\|_{L^{\infty}} \leq N$, for some $N>0$. Then to each $\epsilon>0$ there corresponds a function $\tau \in$ $L^{1}\left(\mathbb{R}^{n-1}\right), \hat{\tau}(0)=1$, such that

$$
\int_{\mathbb{R}^{n}}\left|\left(\tau * f_{1}\right)(x)\right| d x+\cdots+\int_{\mathbb{R}^{n}}\left|\left(\tau * f_{M}\right)(x)\right| d x \leq \int_{\mathbb{R}}\left\|P_{1} f(t)\right\|_{\mathbb{R}^{M}} d t+\epsilon,
$$

([15], pp. 113, 115). This proves the theorem since such functions are dense in $L^{1}\left(\mathbb{R}^{n}, \mathbb{R}^{M}\right)$ as $K$ and $N$ are allowed to vary.

Proof of necessity of the maximal rank condition can now be completed. Since $\mathbb{K}$ commutes with translation, hence with convolution by $\tau,(5.4)$ together with (5.5) ensures that

$$
\left\|P_{\mathbf{1}} f\right\|_{H^{1}} \leq \inf _{\tau}\|\tau *(\mathbb{K} f)\|_{L^{1}}=\int_{\mathbb{R}}\left\|\mathbb{K}_{\mathbf{1}} f(t)\right\|_{\mathbb{R}^{M}} d t
$$

establishing (5.3). This inequality will be applied to a copy of $H^{1}(\mathbb{R})$ in $H^{1}\left(\mathbb{R}, \Lambda_{r}\left(\mathbb{C}^{n-1}\right)\right)$. For any $a, b \in \Lambda_{r}\left(\mathbb{C}^{n-1}\right)$,

$$
h \longrightarrow h_{a b}=(a-b) h-i(a+b) \tilde{h}=a(h-i \tilde{h})-b(h+i \tilde{h})
$$

embeds $H^{1}(\mathbb{R})$ in $H^{1}\left(\mathbb{R}, \Lambda_{r}\left(\mathbb{C}^{n-1}\right)\right)$ so that

$$
\left\|h_{a b}\right\|_{H^{1}}=\|(a-b) h-i(a+b) \tilde{h}\|_{H^{1}} \cong(\|a\|+\|b\|)\|h\|_{H^{1}} .
$$

Now apply $\mathbb{K}_{\mathbf{1}}$ to such functions:

$$
\mathbb{K}_{\mathbf{1}} h_{a b}=(m(\mathbf{1}) a-m(-\mathbf{1}) b) h-i(m(\mathbf{1}) a+m(-\mathbf{1}) b) \tilde{h} .
$$

But if $m(\mathbf{1}) a+m(-\mathbf{1}) b=0$, then

$$
(\|a\|+\|b\|)\|h\|_{H^{1}} \leq \text { const. }\left(\|m(\mathbf{1}) a-m(-\mathbf{1}) b\|_{\mathbb{C}^{M}}\right)\|h\|_{L^{1}}
$$


can occur for all $f \in H^{1}(\mathbb{R})$ only if $a=b=0$. Thus $m(\mathbf{1}) \oplus m(-\mathbf{1})$ must have maximal rank on $T_{r}(\mathbf{1}) \oplus T_{r}(-\mathbf{1})$. After rotation the proof is complete. This proves that if $\mathbb{K}$ characterizes $H_{\rho_{r}}^{1}$ then the rank condition holds.

Proof of sufficiency. We begin with a $\Lambda_{r}\left(\mathbb{C}^{n}\right)$-valued version of Uchiyama's characterization of $H^{1}$.

Lemma $5.6([\mathbf{2 0}])$. Let $\tilde{\mathbb{K}}$ be a standard singular integral operator having symbol $\tilde{m}(\omega)$ mapping $\Lambda_{r}\left(\mathbb{C}^{n}\right)$-valued functions to $\mathbb{C}^{M}$-valued functions. Then $\tilde{\mathbb{K}}$ characterizes $H^{1}\left(\mathbb{R}^{n}, \Lambda_{r}\left(\mathbb{C}^{n}\right)\right)$ if and only if for each $\omega \in \Sigma_{n-1}$,

$$
\operatorname{rank}(\tilde{m}(\omega) \oplus \tilde{m}(-\omega))=2\left(\begin{array}{l}
n \\
r
\end{array}\right) \text {. }
$$

Now suppose that the symbol of $\mathbb{K}$ satisfies $\operatorname{rank}(m(\omega) \oplus m(-\omega))=2\left(\begin{array}{c}n-1 \\ r\end{array}\right)$ as a mapping $(a, b) \rightarrow m(\omega) a+m(-\omega) b$ from $T_{r}(\omega) \oplus T_{r}(-\omega)$ into $\mathbb{C}^{M}$. Let $\mathbb{N}_{r}$ be the system of singular integrals in Example $\left(2.5^{\prime}\right)$ with symbol $n$, and consider the augmented system $\tilde{\mathbb{K}}$ having symbol $\left(\begin{array}{c}m(\omega) \\ n(\omega)\end{array}\right)$. Clearly $\tilde{m}$ satisfies $\operatorname{rank}(\tilde{m}(\omega) \oplus \tilde{m}(-\omega))=2\left(\begin{array}{l}n \\ r\end{array}\right)$ as a mapping on $\mathbb{C}^{M} \oplus \mathbb{C}^{M}$. By (5.6), $\tilde{\mathbb{K}}$ then characterizes $H^{1}\left(\mathbb{R}^{n}, \Lambda_{r}\left(\mathbb{C}^{M}\right)\right)$. Since any $f \in H_{\rho_{r}}^{1}$ lies in the kernel of $\mathbb{N}_{r}$ it follows that $\|f\|_{H_{\rho_{r}}^{1}} \sim\|\tilde{\mathbb{K}} f\|_{L^{1}}=\|\mathbb{K} f\|_{L^{1}}$. Therefore $\mathbb{K}$ characterizes $H_{\rho_{r}}^{1}$. This completes the proof of Theorem A.

\section{Pseudo-inverses.}

Pseudoinverses play a fundamental role in Uchiyama's constructive decomposition. In our setting these are systems of real singular integrals $\mathbb{L}_{\nu}=$ $\left(\mathcal{L}_{\nu_{1}}, \ldots, \mathcal{L}_{\nu_{M}}\right)$

$$
\mathcal{L}_{\nu_{j}} f(x)=\int_{\mathbb{R}^{n}} e^{2 \pi i x \cdot \xi}\left\langle\hat{f}(\xi), \theta_{\nu_{j}}(\xi)\right\rangle d \xi
$$

indexed by elements $\nu=\left(\nu_{1}, \ldots, \nu_{M}\right)$ in the unit sphere $\Sigma_{M-1}$ of $\mathbb{R}^{M}$ and satisfying standard estimates independent of $\nu$; here $\theta_{\nu_{1}}, \ldots, \theta_{\nu_{M}}$ are $\Lambda_{r}(\mathbb{C})$ valued functions on $\Sigma_{n-1}$ extended to homogeneous functions of degree zero on $\mathbb{R}^{n} \backslash\{0\}$. We shall follow the same path, always mindful of the fact that the mapping $m(\omega) \oplus m(-\omega)$ need not be 1-1 everywhere on $\Lambda_{r}\left(\mathbb{C}^{n}\right) \oplus \Lambda_{r}\left(\mathbb{C}^{n}\right)$.

Theorem D. If the mapping $m(\omega) \oplus m(-\omega)$ associated with the symbol of $\mathbb{K}$ has the property that for each $\omega \in \Sigma_{n-1}$

$$
\operatorname{rank}(m(\omega) \oplus m(-\omega))=2\left(\begin{array}{c}
n-1 \\
r
\end{array}\right)
$$

as a mapping from $T_{r}(\omega) \oplus T_{r}(-\omega)$ into $\mathbb{C}^{M}$, then there exists a system of real singular integrals $\mathbb{L}_{\nu}=\left(\mathcal{L}_{\nu_{1}}, \ldots, \mathcal{L}_{\nu_{M}}\right), \nu \in \Sigma_{M-1}$, depending smoothly 
on $\nu$ and such that

$$
\sum_{j} \nu_{j} \mathcal{L}_{\nu_{j}}=0, \quad \mathbb{K}^{*} \circ \mathbb{L}_{\nu}=I
$$

on $H_{\rho_{r}}^{1}\left(\mathbb{R}^{n}\right)$ for each $\nu$.

Smooth dependence i.e., $\mathcal{C}^{\infty}$-dependence, ensures that the standard estimates for $\mathbb{L}_{\nu}$ are uniform in $\nu$.

Clearly the system $\mathbb{L}_{\nu}$ acts as a 'partial' inverse to $\mathbb{K}$ in the sense that it inverts $\mathbb{K}$ on $H_{\rho_{r}}^{1}\left(\mathbb{R}^{n}\right)$, but not necessarily on all of $H^{1}\left(\mathbb{R}^{n}, \Lambda_{r}\right)$, just as every mapping $A$ between finite dimensional spaces admits a partial inverse - the Moore-Penrose pseudo-inverse $A^{+}$of $A$, for instance ([14]). Because $A^{+}$inverts $A$ on the subspace orthogonal to the kernel of $A$, the maximal rank condition on $\mathbb{K}$ will allow us to define $\mathbb{L}_{\nu}$ via its symbol by applying the Moore-Penrose pseudo-inverse construction at each point $\omega$ of $\Sigma_{n-1}$. As we have to ensure that everything can be done smoothly both in $\omega$ and $\nu$, it may be helpful to recall some basic ideas underlying the construction of $A^{+}$from $A$. First Penrose shows that equations

$$
X X^{*} A^{*}=X, \quad X A A^{*}=A^{*}
$$

have a unique solution $X$ which he takes as the 'generalized inverse' $A^{+}$of $A$; of course, if $A$ has an inverse $A^{-1}$ in the usual sense, then $A^{-1}$ obviously satisfies (6.2)(i), so $A^{+}=A^{-1}$ when the latter is well-defined. He then shows that $B A^{*}$ is a solution of $(6.2)(\mathrm{i})$ provided

$$
B A^{*} A A^{*}=A^{*}
$$

and obtains one such $B$ using the Cayley-Hamilton theorem. It is this last construction that we need to show can be done smoothly, though it should be observed that Penrose himself remarked that it could be done continuously ([14], p. 408).

Theorem 6.3. Let $A_{\alpha}$ be a family of $m \times n$ complex matrices varying smoothly with $\alpha$ on some open set $\mathcal{A}$. Then the Moore-Penrose inverse $A_{\alpha}^{+}$of $A_{\alpha}$ varies smoothly with $\alpha$ on any open subset of $\mathcal{A}$ on which the rank of $A_{\alpha}$ remains constant.

Proof. Let

$$
f_{\alpha}(\lambda)=\operatorname{det}\left(A_{\alpha}^{*} A_{\alpha}-\lambda I\right)=a_{0}(\alpha)+a_{1}(\alpha) \lambda+\cdots+a_{n}(\alpha) \lambda^{n}
$$

be the characteristic polynomial of $A_{\alpha}^{*} A_{\alpha}$. The coefficients of $f_{\alpha}$ will be smooth on $\mathcal{A}$ and $A^{*} A$ will be a solution of the polynomial equation $f_{\alpha}(\lambda)=$ 0 . On the other hand, $\operatorname{since} \operatorname{rank}\left(A_{\alpha}\right)=\operatorname{rank}\left(A_{\alpha}^{*} A_{\alpha}\right)$,

$$
f_{\alpha}(\lambda)=\lambda^{n-r}\left(a_{n-r}(\alpha)+\cdots+a_{n}(\alpha) \lambda^{r}\right), \quad\left(r=\operatorname{rank} A_{\alpha}\right) .
$$

Thus

$$
a_{0}(\alpha)=a_{1}(\alpha)=\cdots=a_{n-r-1}(\alpha)=0, \quad a_{n-r}(\alpha) \neq 0
$$


on any open subset of $\mathcal{A}$ on which $A_{\alpha}$ has constant rank $r$, while

$$
\left(A_{\alpha}^{*} A_{\alpha}\right)^{n-r}\left(a_{n-r}(\alpha) I+\cdots+a_{n}(\alpha)\left(A_{\alpha}^{*} A_{\alpha}\right)^{r}\right)=0
$$

on this same subset. Now define $B_{\alpha}$ by

$$
B_{\alpha}=-\frac{1}{a_{n-r}(\alpha)}\left(a_{n-r+1}(\alpha) I+\cdots+a_{n}(\alpha)\left(A_{\alpha}^{*} A_{\alpha}\right)^{r-1}\right) .
$$

Property (6.4) ensures that

$$
B_{\alpha}\left(A_{\alpha}^{*} A_{\alpha}\right)^{n-r+1}=\left(A_{\alpha}^{*} A_{\alpha}\right)^{n-r},
$$

which after succesive cancellation by $A_{\alpha}$ and $A_{\alpha}^{*}$ on the right reduces to

$$
B_{\alpha} A_{\alpha}^{*} A_{\alpha} A_{\alpha}^{*}=A_{\alpha}^{*}
$$

([14], p. 406). This completes the proof.

We can now begin the proof of Theorem D. For each $\nu$ in $\Sigma_{M-1}$ of $\mathbb{R}^{M}$ and $\omega$ in $\Sigma_{n-1}$ define $\mu_{\nu}: \mathbb{C} \oplus \Lambda_{r}\left(\mathbb{C}^{n}\right) \rightarrow \mathbb{C}^{M}$ by

$$
\mu_{\nu}(\omega):(\lambda, a) \longrightarrow \mu_{\nu}(\omega)(\lambda, a)=\lambda \nu+m(\omega) \pi_{T}(\omega) a
$$

where $\pi_{T}(\omega)$ is the orthogonal projection from $\Lambda_{r}\left(\mathbb{C}^{n}\right)$ onto $T_{r}(\omega)$. The first result brings out very clearly how the algebraic condition on the symbol of $\mathbb{K}$ and the fact that $\mathbb{K}$ is real permit use of the Moore-Penrose pseudo-inverse $\mu_{\nu}^{+}(\omega)$ of $\mu_{\nu}(\omega)$.

Theorem 6.8. If the symbol of $\mathbb{K}$ satisfies the maximal rank condition of Theorem A, then

$$
\operatorname{ker}\left(\mu_{\nu}(\omega)\right)=\left\{(0, b): b \in N_{r}(\omega)\right\}, \quad \operatorname{rank}\left(\mu_{\nu}(\omega)\right)=1+\left(\begin{array}{c}
n-1 \\
r
\end{array}\right)
$$

for each $(\nu, \omega) \in \Sigma_{M-1} \times \Sigma_{n-1}$. In particular, $\mu_{\nu}^{+}(\omega)$ is smooth as a function on $\Sigma_{M-1} \times \Sigma_{n-1}$ and $\mu_{\nu}^{+}(\omega) \mu_{\nu}(\omega)=I$ on $\mathbb{C} \oplus T_{r}(\omega)$.

Proof. Obviously,

$$
\operatorname{ker}\left(\mu_{\nu}(\omega)\right) \supseteq\left\{(0, b): b \in N_{r}(\omega)\right\} .
$$

The reverse inclusion will follow from the restrictions on the symbol of $\mathbb{K}$. Suppose $(\lambda, a)$ lies in the kernel of $\mu_{\nu}(\omega)$ and let $a=a_{0}+a_{1}$ be the decomposition of an element of $\Lambda_{r}\left(\mathbb{C}^{n}\right)$ into its respective normal and tangential components $a_{0}, a_{1}$ at $\omega$. Then

$$
\mu_{\nu}(\omega)(\lambda, a)=\lambda \nu+m(\omega) a_{1}=0,
$$

and so

$$
m(\omega) a_{1}=0 \quad(\lambda=0), \quad m(\omega)\left(a_{1} / \lambda\right)=-\nu \quad(\lambda \neq 0) .
$$


But the maximal rank condition ensures that $m(\omega)$ is $1-1$ on $T_{r}(\omega)$; consequently, $a_{1}=0$ if $\lambda=0$. If $\lambda \neq 0$ on the other hand, set $b=a_{1} / \lambda$. Then $\bar{b} \in T_{r}(-\omega)$ and, because $\mathbb{K}$ is real,

$$
\overline{m(\omega)(b)}=m(-\omega)(\bar{b}) \in \Sigma_{M-1} ;
$$

in this case, $m(\omega) b+m(-\omega)(-\bar{b})=0$, contradicting the maximal rank condition. Hence $\operatorname{ker}\left(\mu_{\nu}(\omega)\right) \subseteq\left\{(0, b): b \in N_{r}(\omega)\right\}$. The remainder of the theorem now follows immediately from (6.3) since $\operatorname{dim}_{\mathbb{C}}\left(T_{r}(\omega)\right)=\left(\begin{array}{c}n-1 \\ r\end{array}\right)$.

In view of (6.8), therefore, there exist

$$
\gamma=\left(\gamma_{1}, \ldots, \gamma_{M}\right), \quad \theta(\nu, \omega)=\left(\theta_{\nu_{1}}(\omega), \ldots, \theta_{\nu_{M}}(\omega)\right)
$$

with $\gamma \in \mathbb{C}^{M}$ and $\theta=\theta(\nu, \omega)$ a $\mathcal{C}^{\infty}$-function $\Sigma_{M-1} \times \Sigma_{n-1} \rightarrow \mathbb{C}^{M} \otimes \Lambda_{r}\left(\mathbb{C}^{n}\right)$ such that

$$
\mu_{\nu}^{+}(\omega): \beta=\left(\beta_{1}, \ldots, \beta_{M}\right) \longrightarrow\left(\langle\beta, \gamma\rangle, \sum_{j} \beta_{j} \theta_{\nu_{j}}(\omega)\right) .
$$

Thus on $\mathbb{C} \oplus T_{r}(\omega)$,

$$
\begin{aligned}
\mu_{\nu}^{+}(\omega) \mu_{\nu}(\omega):(\lambda, a) & \longrightarrow(\lambda\langle\nu, \gamma\rangle+\langle m(\omega) a, \gamma\rangle, \\
& \left.\lambda \sum_{j} \nu_{j} \theta_{\nu_{j}}(\omega)+\sum_{j}\left\langle a, m_{j}(\omega)\right\rangle \theta_{\nu_{j}}(\omega)\right)=(\lambda, a) .
\end{aligned}
$$

But this can happen only if

$$
\sum_{j} \nu_{j} \theta_{\nu_{j}}(\omega) \in N_{r}(\omega), \quad \sum_{j}\left\langle a, m_{j}(\omega)\right\rangle \theta_{\nu_{j}}(\omega)=a
$$

for all $a \in T_{r}(\omega)$. These properties of $\left(\theta_{\nu_{1}}(\omega), \ldots, \theta_{\nu_{M}}(\omega)\right)$ ensure that the singular integrals defined by (6.1) have the properties required of the system $\mathbb{L}_{\nu}=\left(\mathcal{L}_{\nu_{1}}, \ldots, \mathcal{L}_{\nu_{M}}\right)$ in Theorem D, completing the proof of that theorem.

\section{Divergence-free $r$-form wavelets.}

In this section we present the construction of the $r$-form wavelets described in Theorem C (cf. [11]). The first step will be the proof of the following result.

Lemma 7.1. For each $r, 1 \leq r \leq n$, there is a bi-orthogonal family $\left\{\left(\gamma^{\epsilon, \alpha}, \eta^{\epsilon, \alpha}\right): \epsilon \in E^{*}, \alpha \in \mathcal{P}_{r}\right\}$ of compactly supported $r$-form wavelets so that each $f$ in $L^{2}\left(\mathbb{R}^{n}, \Lambda_{r}\right)$ admits a wavelet expansion

$$
f(x)=\sum_{\alpha \in \mathcal{P}_{r}} \sum_{\epsilon, Q}\left\langle f, \eta_{Q}^{\epsilon, \alpha}\right\rangle \gamma_{Q}^{\epsilon, \alpha}(x)
$$


which commutes with interior differentiation in the sense that

$$
\begin{aligned}
& \mu_{k}^{*} \frac{\partial}{\partial x_{k}}\left(\sum_{Q \in \mathcal{Q}_{m}}\left\langle f, \eta_{Q}^{\epsilon, \alpha}\right\rangle \gamma_{Q}^{\epsilon, \alpha}(x)\right) \\
& =\sum_{Q \in \mathcal{Q}_{m}}\left\langle\mu_{k}^{*} D_{k} f, \eta_{Q}^{\epsilon, \alpha \backslash k}\right\rangle \gamma_{Q}^{\epsilon, \alpha \backslash k}(x) \quad(k \in \alpha)
\end{aligned}
$$

holds for each $\epsilon, \alpha, k$ and $m$ where $\alpha \backslash k$ denotes the $(r-1)$-tuple obtained by omitting $k$ from the $r$-tuple $\alpha$.

Note that the sum in (7.2)(i) is taken over the set $\mathcal{Q}$ of all dyadic cubes, while the sums in (7.2)(ii) are taken over the set $\mathcal{Q}_{m}$ of dyadic cubes having side-length $l(Q)=2^{-m}$. The divergence-free wavelets $\psi_{\epsilon, \alpha}$ will arise as suitable linear combinations of the $\gamma_{\epsilon, \alpha}$ - this will be the second step in the construction.

Let $\phi=\phi(s), s \in \mathbb{R}$, be a compactly supported real-valued scaling function on $\mathbb{R}$ and $\psi=\psi(s)$ the associated wavelet. The usual translates and dilates of $\psi$ produce an orthonormal basis for $L^{2}(\mathbb{R})$, while tensor products of $\{\phi, \psi\}$ generate wavelets on $\mathbb{R}^{n}([\mathbf{1 3}]$, p. 79): For each $n$-tuple $\epsilon=\left(\epsilon_{1}, \ldots, \epsilon_{n}\right)$ in $E^{*}$ and $x=\left(x_{1}, \ldots, x_{n}\right)$ in $\mathbb{R}^{n}$ set

$$
\psi_{\epsilon}(x)=\prod_{j=1}^{n}\left(\epsilon_{j} \phi\left(x_{j}\right)+\left(1-\epsilon_{j}\right) \psi\left(x_{j}\right)\right) .
$$

Then translates and dilates of the family $\left\{\psi_{\epsilon}: \epsilon \in E^{*}\right\}$ provide an orthonormal basis for $L^{2}\left(\mathbb{R}^{n}\right)$.

The fundamental idea of Lemarié was to use multi-resolution analysis for the bi-orthogonal case $([2]$, p. 128) to derive bi-orthogonal pairs of scaling functions $\left\{\phi_{+}, \phi_{-}\right\}$and wavelets $\left\{\psi_{+}, \psi_{-}\right\}$from the initial choice of $(\phi, \psi)$ by exploiting Viete's formula

$$
\frac{\sin \pi \sigma}{\pi \sigma}=\prod_{k=1}^{\infty} \cos \left(\frac{1}{2^{k}} \pi \sigma\right) \quad(\sigma \in \mathbb{R}),
$$

in conjunction with the difference operators

$$
\Delta_{+}: f(s) \longrightarrow f(s+1)-f(s), \quad \Delta_{-}: f(s) \longrightarrow f(s)-f(s-1)
$$

for functions on $\mathbb{R}$. Property (7.4) ensures that

$$
\hat{\phi}_{+}(\sigma)=e^{-\pi i \sigma}\left(\frac{\sin \pi \sigma}{\pi \sigma}\right) \hat{\phi}(\sigma), \quad \hat{\phi}_{-}(\sigma)=e^{-\pi i \sigma}\left(\frac{\pi \sigma}{\sin \pi \sigma}\right) \hat{\phi}(\sigma)
$$


define new scaling functions $\left\{\phi_{+}, \phi_{-}\right\}$which will have compact support provided $\phi$ is sufficiently smooth, while translates and dilates of the corresponding pair of compactly supported wavelets $\left\{\left(\psi_{+}, \psi_{-}\right)\right\}$,

$$
\hat{\psi}_{+}(\sigma)=\left(\frac{2 i}{\pi \sigma}\right) \hat{\psi}(\sigma), \quad \hat{\psi}_{-}(\sigma)=\left(\frac{\pi i \sigma}{2}\right) \hat{\psi}(\sigma)
$$

provide a bi-orthogonal basis for $L^{2}(\mathbb{R})$. On the other hand, since

$$
(D f)^{\wedge}(\sigma)=e^{-\pi i \xi}\left(\frac{\pi \sigma}{\sin \pi \sigma}\right)\left(\Delta_{+} f\right)^{\wedge}(\sigma)=e^{\pi i \xi}\left(\frac{\pi \sigma}{\sin \pi \sigma}\right)\left(\Delta_{-} f\right)^{\wedge}(\sigma),
$$

property (7.6) ensures that

$$
D \phi_{+}=\Delta_{-} \phi, \quad D \phi=\Delta_{+} \phi_{-}, \quad D \psi_{+}=-4 \psi, \quad D \psi=4 \psi_{-} .
$$

Finally, translates and dilates of the family $\left\{\left(\psi_{\epsilon,+}, \psi_{\epsilon,-}\right): \epsilon \in E^{*}\right\}$ of tensor products

$$
\begin{aligned}
& \psi_{\epsilon,+}(x)=\prod_{j=1}^{n}\left(\epsilon_{j} \psi_{+}\left(x_{j}\right)+\left(1-\epsilon_{j}\right) \phi_{+}\left(x_{j}\right)\right), \\
& \psi_{\epsilon,-}(x)=\prod_{j=1}^{n}\left(\epsilon_{j} \psi_{-}\left(x_{j}\right)+\left(1-\epsilon_{j}\right) \phi_{-}\left(x_{j}\right)\right)
\end{aligned}
$$

generate a bi-orthogonal wavelet basis for $L^{2}\left(\mathbb{R}^{n}\right)$.

The bi-orthogonal wavelets in (7.1), and hence those needed for Theorem $\mathrm{B}$, will be obtained by the same tensor product construction as before, but in an important sense to be made precise shortly, the construction will produce families intermediate to those in (7.3) and (7.9).

Proof of Lemma 7.1. For each $\epsilon \in E^{*}$ and $\alpha \in \mathcal{P}_{r}$ set

$$
\gamma_{\epsilon, \alpha}(x)=\prod_{j \in \alpha}\left(\epsilon_{j} \psi_{+}\left(x_{j}\right)+\left(1-\epsilon_{j}\right) \phi_{+}\left(x_{j}\right)\right) \prod_{j \notin \alpha}\left(\epsilon_{j} \psi\left(x_{j}\right)+\left(1-\epsilon_{j}\right) \phi\left(x_{j}\right)\right)
$$

and

$$
\eta_{\epsilon, \alpha}(x)=\prod_{j \in \alpha}\left(\epsilon_{j} \psi_{-}\left(x_{j}\right)+\left(1-\epsilon_{j}\right) \phi_{-}\left(x_{j}\right)\right) \prod_{j \notin \alpha}\left(\epsilon_{j} \psi\left(x_{j}\right)+\left(1-\epsilon_{j}\right) \phi\left(x_{j}\right)\right) .
$$

Notice that $\gamma_{\epsilon, \alpha}=\eta_{\epsilon, \alpha}=\psi_{\epsilon}$ when $\alpha$ is the empty set, i.e., $r=0$, whereas $\gamma_{\epsilon, \alpha}=\psi_{\epsilon,+}$ and $\eta_{\epsilon, \alpha}=\psi_{\epsilon,-}$ when $\alpha$ is the $n$-tuple $(1,2, \ldots, n)$, i.e., when $r=n$. Since each of the latter two families generates a bi-orthogonal basis for $L^{2}\left(\mathbb{R}^{n}\right)$ as $\epsilon$ varies over $E^{*}$, so the family $\left\{\left(\gamma_{\epsilon, \alpha}, \eta_{\epsilon, \alpha}\right): \epsilon \in E^{*}\right\}$ generates a bi-orthogonal basis for $L^{2}\left(\mathbb{R}^{n}\right)$ for each fixed $\alpha \in \mathcal{P}_{r}$. To convert these realvalued functions into $r$-forms set

$$
\gamma^{\epsilon, \alpha}(x)=\gamma_{\epsilon, \alpha}(x) e_{\alpha}, \quad \eta^{\epsilon, \alpha}(x)=\eta_{\epsilon, \alpha}(x) e_{\alpha}
$$


with $\left\{e_{\alpha}: \alpha \in \mathcal{P}_{r}\right\}$ the orthonormal basis for $\Lambda_{r}$ of wedge products (3.2). By construction therefore, the translates and dilates of $\left\{\left(\gamma^{\epsilon, \alpha}, \eta^{\epsilon, \alpha}\right): \epsilon \in E^{*}, \alpha \in\right.$ $\left.\mathcal{P}_{r}\right\}$ provide a bi-orthogonal basis for $L^{2}\left(\mathbb{R}^{n}, \Lambda_{r}\right)$, establishing (7.2)(i).

On the other hand, because of (7.8),

$$
\mu_{k}^{*} \frac{\partial \gamma^{\epsilon, \alpha}}{\partial x_{k}}=\left\langle\mu_{k}^{*}\left(e_{\alpha}\right), e_{\alpha \backslash k}\right\rangle\left\{\left(1-\epsilon_{k}\right) \Delta_{-}(k)-4 \epsilon_{k}\right\} \gamma^{\epsilon, \alpha \backslash k} \quad(k \in \alpha)
$$

while

$$
\text { (7.11)(ii) } \mu_{k} \frac{\partial \eta^{\epsilon, \alpha \backslash k}}{\partial x_{k}}=\left\langle\mu_{k}\left(e_{\alpha \backslash k}\right), e_{\alpha}\right\rangle\left\{\left(1-\epsilon_{k}\right) \Delta_{+}(k)+4 \epsilon_{k}\right\} \eta^{\epsilon, \alpha}
$$

where

$$
\Delta_{+}(k): f(x) \longrightarrow f\left(x+e_{k}\right)-f(x), \quad \Delta_{-}(k): f(x) \longrightarrow f(x)-f\left(x-e_{k}\right)
$$

are the difference operators $\Delta_{ \pm}$applied now to the $x_{k}$ variable of a function on $\mathbb{R}^{n}$. Property (7.2)(ii) is now easily established. When $\epsilon_{k}=0$, for instance, summation by parts ensures that

$$
\mu_{k}^{*} \frac{\partial}{\partial x_{k}}\left(\sum_{Q \in \mathcal{Q}_{m}}\left\langle f, \eta_{Q}^{\epsilon, \alpha}\right\rangle \gamma_{Q}^{\epsilon, \alpha}\right)=-\sum_{Q \in \mathcal{Q}_{m}}\left\langle f, \mu_{k} D_{k} \eta_{Q}^{\epsilon, \alpha \backslash k}\right\rangle \gamma_{Q}^{\epsilon, \alpha \backslash k}
$$

from which (7.2)(ii) follows after integrating by parts; whereas when $\epsilon_{k}=1$,

$$
\mu_{k}^{*} \frac{\partial}{\partial x_{k}}\left(\left\langle f, \eta_{Q}^{\epsilon, \alpha}\right\rangle \gamma_{Q}^{\epsilon, \alpha}\right)=-\left\langle f, \mu_{k} D_{k} \eta_{Q}^{\epsilon, \alpha \backslash k}\right\rangle \gamma_{Q}^{\epsilon, \alpha \backslash k}
$$

and again (7.2)(ii) follows after integrating by parts. This establishes (7.1).

Now we come to the final step in the proof of Theorem C, replacing of $\left\{\gamma^{\epsilon, \alpha}: \epsilon \in E^{*}, \alpha \in \mathcal{P}_{r}\right\}$ by a family $\left\{\psi^{\epsilon, \alpha}: \epsilon \in E^{*}, \alpha \in \mathcal{T}_{r}\right\}$ of divergence-free wavelets so that

$$
f \longrightarrow \sum_{\epsilon, Q} \sum_{\alpha \in \mathcal{T}_{r}}\left\langle f, \eta_{Q}^{\epsilon, \alpha}\right\rangle \psi_{Q}^{\epsilon, \alpha}(x)
$$

is the Hodge projection. Recall that $\mathcal{P}_{r}=\mathcal{T}_{r} \cup \mathcal{N}_{r}$ where

$$
\mathcal{T}_{r}=\left\{\alpha \in \mathcal{P}_{r}: i_{\epsilon} \notin \alpha\right\}, \quad \mathcal{N}_{r}=\left\{\beta \in \mathcal{P}_{r}: i_{\epsilon} \in \beta\right\}, \quad i_{\epsilon}=\min \left\{k: \epsilon_{k}=1\right\},
$$

and set

$$
\psi^{\epsilon, \alpha}(x)=-\frac{1}{4}\left\langle\mu_{i_{\epsilon}}^{*}\left(e_{a}\right), e_{\alpha}\right\rangle d^{*} \gamma^{\epsilon, a} \quad\left\{\begin{array}{l}
\alpha \in \mathcal{T}_{r}, \\
a=\alpha \cup i_{\epsilon} .
\end{array}\right.
$$

Clearly each $\psi^{\epsilon, \alpha}$ is a divergence-free $r$-form; in addition, at the risk of forcing $\psi^{\epsilon, \alpha}, \eta^{\epsilon, \alpha}$ to have large compact support, we can assume that both functions have continuous derivatives and vanishing moments up to some prescribed order - this can be achieved by choosing the original $\phi$ to be a compactly supported scaling function having sufficiently many derivatives 
and scaling filter with sufficiently many zeroes $([\mathbf{1 3}]$, p. 93). On the other hand, by (7.11) and the choice $a=\alpha \cup i_{\epsilon}$,

$$
\begin{aligned}
\psi^{\epsilon, \alpha} & =\gamma^{\epsilon, \alpha}(x)-\frac{1}{4}\left\langle\mu_{i_{\epsilon}}^{*}\left(e_{a}\right), e_{\alpha}\right\rangle \sum_{k \in \alpha} \mu_{k}^{*} \frac{\partial \gamma^{\epsilon, a}}{\partial x_{k}} \\
& =\gamma^{\epsilon, \alpha}+\left\langle\mu_{i_{\epsilon}}^{*}\left(e_{a}\right), e_{\alpha}\right\rangle \sum_{k \in \alpha}\left\langle\mu_{k}^{*}\left(e_{a}\right), e_{a \backslash k}\right\rangle \gamma^{\epsilon, a \backslash k}
\end{aligned}
$$

since $\epsilon_{i_{\epsilon}}=1$. Since $a \backslash k \in \mathcal{N}_{r}$ when $k \in \alpha,\left\{\left(\psi^{\epsilon, \alpha}, \eta^{\epsilon, \alpha}\right): \epsilon \in E^{*}, \alpha \in \mathcal{T}_{r}\right\}$ is a bi-orthogonal family in the sense that

$$
\left\langle\psi_{Q}^{\epsilon, \alpha}, \eta_{Q^{\prime}}^{\epsilon^{\prime}, \alpha^{\prime}}\right\rangle=\delta_{\epsilon \epsilon^{\prime}} \delta_{\alpha \alpha^{\prime}} \delta_{Q Q^{\prime}} \quad\left(\alpha, \alpha^{\prime} \in \mathcal{T}_{r}\right) .
$$

This establishes part (i) of Theorem $\mathrm{C}$, leaving only the proof of the projection property (ii). Now certainly (7.12) maps $L^{2}\left(\mathbb{R}^{n}, \Lambda_{r}\right)$ into $H_{\rho_{r}}^{2}\left(\mathbb{R}^{n}\right)$ as each $\psi_{Q}^{\epsilon, \alpha}(x)$ is massless. To complete the proof, therefore, we have to show that each $f$ in $H_{\rho_{r}}^{2}\left(\mathbb{R}^{n}\right)$ can be represented as

$$
f(x)=\sum_{\epsilon, Q} \sum_{\alpha \in \mathcal{T}_{r}}\left\langle f, \eta_{Q}^{\epsilon, \alpha}\right\rangle \psi_{Q}^{\epsilon, \alpha}(x) .
$$

But every $f$ has a wavelet expansion

$$
f(x)=\sum_{\beta \in \mathcal{P}_{r}} \sum_{\epsilon, Q}\left\langle f, \eta_{Q}^{\epsilon, \beta}\right\rangle \gamma_{Q}^{\epsilon, \beta}(x),
$$

so it is enough to show that the expansions in (7.14) coincide on $H_{\rho_{r}}^{2}\left(\mathbb{R}^{n}\right)$. Let us assume for the moment that the following technical result has been established.

Lemma 7.15. For each $\epsilon$ in $E^{*}$ the identity

$$
\begin{aligned}
& \mu_{i_{\epsilon}}^{*} \frac{\partial}{\partial x_{i_{\epsilon}}}\left(\sum_{\beta \in \mathcal{P}_{r}} \sum_{Q \in \mathcal{Q}_{m}}\left\langle f, \eta_{Q}^{\epsilon, \beta}\right\rangle \gamma_{Q}^{\epsilon, \beta}-\sum_{\alpha \in \mathcal{T}_{r}} \sum_{Q \in \mathcal{Q}_{m}}\left\langle f, \eta_{Q}^{\epsilon, \alpha}\right\rangle \psi_{Q}^{\epsilon, \alpha}\right) \\
& =\sum_{\delta \in \mathcal{T}_{r-1}} \sum_{Q \in \mathcal{Q}_{m}}\left\langle d^{*} f, \eta_{Q}^{\epsilon, \delta}\right\rangle \gamma_{Q}^{\epsilon, \delta}
\end{aligned}
$$

holds for all $f$ in $L^{2}\left(\mathbb{R}^{n}, \Lambda_{r}\right)$ and all levels of resolution $m$.

The expression $F$ in parentheses on the left hand side of (7.15) has the form $F=\sum_{\delta \in \mathcal{N}_{r}} \sum_{Q \in \mathcal{Q}_{m}} c_{Q}^{\epsilon, \delta} \gamma_{Q}^{\epsilon, \delta}$ since, by the definition of $\psi_{Q}^{\epsilon, \alpha}$, the $\mathcal{T}_{r}$ terms cancel. But $\delta \in \mathcal{N}_{r}$ implies $i_{\epsilon} \in \delta$ so that $\mu_{i_{\epsilon}}^{*} \frac{\partial}{\partial x_{i_{\epsilon}}} F=0$ implies $F=0$. The expansions in (7.14) thus coincide on $L^{2}$-massless $r$-forms completing the proof of Theorem C. 
Proof of Lemma 7.15. In view of the definition of $\mathcal{T}_{r}$ and $\mathcal{N}_{r}$,

$$
\begin{gathered}
\mu_{i_{\epsilon}}^{*} \frac{\partial}{\partial x_{i_{\epsilon}}}\left(\sum_{\beta \in \mathcal{P}_{r}} \sum_{Q \in \mathcal{Q}_{m}}\left\langle f, \eta_{Q}^{\epsilon, \beta}\right\rangle \gamma_{Q}^{\epsilon, \beta}\right) \\
=\sum_{\delta \in \mathcal{T}_{r-1}} \sum_{Q \in \mathcal{Q}_{m}}\left\langle\mu_{i_{\epsilon}}^{*} D_{i_{\epsilon}} f, \eta_{Q}^{\epsilon, \delta}\right\rangle \gamma_{Q}^{\epsilon, \delta} .
\end{gathered}
$$

On the other hand, for any $\alpha \in \mathcal{T}_{r}$,

$$
\begin{aligned}
& \sum_{Q \in \mathcal{Q}_{m}}\left\langle f, \eta_{Q}^{\epsilon, \alpha}\right\rangle \psi_{Q}^{\epsilon, \alpha} \\
= & -\frac{1}{4}\left\langle\mu_{i_{\epsilon}}^{*}\left(e_{a}\right), e_{\alpha}\right\rangle \sum_{Q \in \mathcal{Q}_{m}}\left\langle f, \eta_{Q}^{\epsilon, \alpha}\right\rangle d^{*} \gamma_{Q}^{\epsilon, a} \\
= & \sum_{Q \in \mathcal{Q}_{m}}\left\langle f, \eta_{Q}^{\epsilon, \alpha}\right\rangle \gamma_{Q}^{\epsilon, \alpha} \pm \frac{1}{4}\left\langle\mu_{i_{\epsilon}}^{*}\left(e_{a}\right), e_{\alpha}\right\rangle \sum_{k \in \alpha}\left(\sum_{Q \in \mathcal{Q}_{m}}\left\langle\mu_{k}^{*} D_{k} f, \eta_{Q}^{\epsilon, \alpha \backslash k}\right\rangle \gamma_{Q}^{\epsilon, a \backslash k}\right)
\end{aligned}
$$

where ' \pm ' depends on whether $k<i_{\epsilon}$. Consequently,

$$
\mu_{i_{\epsilon}}^{*} \frac{\partial}{\partial x_{i_{\epsilon}}}\left(\sum_{Q \in \mathcal{Q}_{m}}\left\langle f, \eta_{Q}^{\epsilon, \alpha}\right\rangle \psi_{Q}^{\epsilon, \alpha}\right)=-\sum_{k \in \alpha}\left(\sum_{Q \in \mathcal{Q}_{m}}\left\langle\mu_{k}^{*} D_{k} f, \eta_{Q}^{\epsilon, \alpha \backslash k}\right\rangle \gamma_{Q}^{\epsilon, \alpha \backslash k}\right) .
$$

The identity now follows, completing the proof.

The usefulness of linking wavelet constructions with the geometry of Euclidean space becomes evident in the proof of Theorem 4.8, the only step remaining to complete the proof of Theorem A and all ancillary results.

Proof of Theorem 4.8. We prove first that

$$
P_{\mathbf{1}}: f \longrightarrow P_{\mathbf{1}} f(t)=\int_{\mathbb{R}^{n-1}} f(y+t \mathbf{1}) d y
$$

maps $f \in H_{\rho_{r}}^{1}\left(\mathbb{R}^{n}\right)$ into $H^{1}\left(\mathbb{R}, \Lambda_{r}\left(\mathbb{R}^{n-1}\right)\right)$. Now each $f \in H_{\rho_{r}}^{1}\left(\mathbb{R}^{n}\right)$ has a wavelet expansion

$$
f(x)=\sum_{\epsilon \in E^{*}, \alpha \in \mathcal{T}_{r}} \sum_{k, m} 2^{m n} a_{k, m}^{\epsilon, \alpha} \psi^{\epsilon, \alpha}\left(2^{m} x-k\right) \quad\left(k \in \mathbb{Z}^{n}, m \in \mathbb{Z}\right)
$$

where

$$
a_{k, m}^{\epsilon, \alpha}=\int_{\mathbb{R}^{n}}\left\langle f(y), \eta^{\epsilon, \alpha}\left(2^{m} y-k\right)\right\rangle d y
$$

and

$$
\|f\|_{H^{1}} \cong \int_{\mathbb{R}^{n}}\left(\sum_{k, m}\left|2^{m n} a_{k, m}^{\epsilon, \alpha}\right|^{2}\left\|\psi^{\epsilon, \alpha}\left(2^{m} x-k\right)\right\|_{\Lambda_{r}}^{2}\right)^{1 / 2} d x
$$


But by construction, $P_{\mathbf{1}}\left(\gamma^{\epsilon, \alpha}\right)=0$ unless $\epsilon=(0, \ldots, 0,1)$, while

$$
P_{\mathbf{1}}\left(\gamma^{\epsilon, \alpha}\right)(t)=\psi(t) e_{\alpha} \quad(\epsilon=(0, \ldots, 0,1)) .
$$

Thus

$$
P_{\mathbf{1}}: \psi^{\epsilon, \alpha} \longrightarrow \psi(t) e_{\alpha}+\int_{\mathbb{R}^{n-1}}(.) d y
$$

where the integrand consists of partial derivatives of compactly supported functions. Consequently, (7.16) remains true with $\gamma^{\epsilon, \alpha}$ replaced by $\psi^{\epsilon, \alpha}$ (cf. (7.13)), and so $P_{1} f$ has a wavelet expansion

$$
P_{\mathbf{1}} f(t)=\sum_{\alpha \in \mathcal{T}_{r}} \sum_{l, m} 2^{m} b_{l, m}^{\alpha} \psi\left(2^{m} t-l\right) e_{\alpha} \quad(\epsilon=(0, \ldots, 0,1))
$$

with coefficients

$$
b_{l, m}^{\alpha}=\sum_{K \in \mathbb{Z}^{n-1}} a_{K, l, m}^{\epsilon, \alpha}
$$

and the same restriction on $\epsilon$. A simple application of Hölder's inequality now shows that

$$
\int_{\mathbb{R}}\left(\sum_{l, m}\left|2^{m} b_{l, m}^{\alpha} \psi\left(2^{m} t-l\right)\right|^{2}\right)^{1 / 2} d t \leq \text { const. }\|f\|_{H^{1}}
$$

for each $\alpha \in \mathcal{T}_{r}$. Thus $P_{\mathbf{1}} f$ is a function in $H^{1}\left(\mathbb{R}, \Lambda_{r}\left(\mathbb{R}^{n-1}\right)\right)$ since the wedge products $\left\{e_{\alpha}: \alpha \in \mathcal{T}_{r}\right\}$ form an orthonormal basis for $\Lambda_{r}\left(\mathbb{R}^{n-1}\right)$; in addition

$$
\left\|P_{1} f\right\|_{H^{1}} \leq \text { const. }\|f\|_{H^{1}} \quad\left(f \in H_{\rho_{r}}^{1}\right) .
$$

To prove that $P_{\mathbf{1}}$ maps $f \in H_{\rho_{r}}^{1}\left(\mathbb{R}^{n}\right)$ onto $H^{1}\left(\mathbb{R}, \Lambda_{r}\left(\mathbb{R}^{n-1}\right)\right)$ we use the atomic decomposition for $H^{1}$-spaces and show first that $P_{\mathbf{1}}$ maps $H^{1}\left(\mathbb{R}^{n}\right)$ onto $H^{1}(\mathbb{R})$. It is enough to show that each atom $a_{I}$ having support in the interval $I=[c, d]$, say, has an atomic pre-image $A_{I^{\prime}}$. For each $x=$ $\left(x_{1}, \ldots, x_{n}\right) \in \mathbb{R}^{n}$ set

$$
A_{I^{\prime}}(x)=\frac{1}{|I|^{n-1}} \begin{cases}a_{I}\left(x_{n}\right), & c \leq x_{j} \leq d, \\ 0, & \text { otherwise. }\end{cases}
$$

Clearly this defines an atom on $\mathbb{R}^{n}$ having support in the cube $I^{\prime}=\left\{x \in \mathbb{R}^{n}\right.$ : $\left.c \leq x_{j} \leq d\right\}$ and such that $P_{\mathbf{1}}\left(A_{I^{\prime}}\right)=a_{I}$. Now fix $f \in H^{1}\left(\mathbb{R}, \Lambda_{r}\left(\mathbb{R}^{n-1}\right)\right)$; it will have a pre-image $g \in H^{1}\left(\mathbb{R}^{n}, T_{r}(\mathbf{1})\right)$ since $T_{r}(\mathbf{1})=\Lambda_{r}\left(\mathbb{R}^{n-1}\right)$. But then $F=\left(\mathcal{R}^{*} \mathcal{R}\right) g$ is a function in $H_{\rho_{r}}^{1}\left(\mathbb{R}^{n}\right)$ such that

$$
P_{\mathbf{1}} F=P_{\mathbf{1}}\left(\mathcal{R}^{*} \mathcal{R} g\right)=P_{\mathbf{1}} g=f,
$$

establishing the onto property. This, finally, completes the proof.

Acknowledgement. The authors would like to both referees for several helpful suggestions. 


\section{References}

[1] M. Christ and D. Geller, Singular integral characterizations of Hardy spaces on homogeneous groups, Duke Math. J., 51 (1984), 547-598.

[2] A. Cohen, Biorthogonal wavelets, in 'Wavelets: A Tutorial in Theory and Applications,' C.K. Chui ed., pp. 123-152, Academic Press, San Diego, 1992.

[3] I. Daubechies, Ten Lectures on Wavelets, CBMS-NSF Regional Conference Series, SIAM, Philadelphia, 1992.

[4] C. Fefferman, Harmonic analysis and $H^{p}$-spaces, in 'Studies in Harmonic Analysis,' J. M. Ash, ed., pp. 38-75, MAA, 1976.

[5] C. Fefferman and E. Stein, $H^{p}$ spaces of several variables, Acta Math., 129 (1972), 137-193.

[6] A. Gandulfo, J. Garcia-Cuerva and M. Taibleson, Conjugate system characterization of $H^{1}$ : counter-examples for the Euclidean plane and local fields, Bull. Amer. Math. Soc., 82 (1976), 83-85.

[7] J.E. Gilbert, J.A. Hogan and J.D. Lakey, Frame decompositions of form-valued Hardy spaces, in 'Clifford Algebras in Analysis and Related Topics,' CRC Press, Boca Raton, (1995), 239-259.

[8] _ Atomic decomposition of divergence-free Hardy spaces, Math. Moravica, Special volume, Proc. IWAA (1997), 33-52.

[9] J.E. Gilbert and M.A.M. Murray, Clifford algebras and Dirac operators in harmonic analysis, Cambridge University Press, Cambridge, 1991.

[10] S. Janson, Characterization of $H^{1}$ by singular integral transforms on martingales and $\mathbb{R}^{n}$, Math. Scand., 41 (1977), 140-152.

[11] P.G. Lemarié-Rieusset, Ondelettes vecteurs á divergence nulle, C.R. Acad. Sci. Paris, 313 (1991), 213-216.

[12] A. McIntosh, Clifford algebras, Fourier theory, Singular integrals, and Harmonic functions in Lipschitz domains, in 'Clifford Algebras in Analysis and Related Topics,' CRC Press, Boca Raton, (1995), 33-87.

[13] Y. Meyer, Ondelettes et operateurs, I, II, III, Hermann ed., Paris, 1990.

[14] R. Penrose, A generalized inverse for matrices, Proc. Camb. Phil. Soc., 51 (1955), 406-413.

[15] H. Reiter, Classical Harmonic Analysis and Locally Compact Groups, Oxford Univ. Press, Oxford, 1968.

[16] E.M. Stein, Harmonic Analysis, Real variable methods, orthogonality, and oscillatory integrals, Princeton University Press, Princeton, N.J., 1993.

[17] E.M. Stein and G. Weiss, Introduction to Fourier analysis on Euclidean Spaces, Princeton University Press, Princeton, N.J., 1970.

[18] _ Generalization of the Cauchy-Riemann equations and representations of the rotation group, Amer. J. Math., 90 (1968), 163-196.

[19] A. Uchiyama, A constructive proof of the Fefferman-Stein decomposition of BMO $\left(\mathbb{R}^{n}\right)$, Acta Math., 148 (1982), 215-241. 
$[20]$ , Private communication.

Received December 15, 1996 and revised February 23, 1999. The first and the third authors were supported in part by NSF grant DMS 93-07655. The second author was supported by Australian Research Council.

The University of Texas at Austin

Austin TX 78712-1082

E-mail address: gilbert@math.utexas.edu

MACQUARIE UNIVERSITY

NSW 2109

Australia

E-mail address: jeffh@mpce.mq.edu.au

New Mexico State University

Las Cruces, NM 88003-8001

E-mail address: jlakey@nmsu.edu 